\title{
Synthesis of Pyroglutamic Acid Derivatives via Double Michael Reactions of Alkynones
}

\author{
Myriam Scansetti, ${ }^{\dagger}$ Xiangping Hu, ${ }^{\dagger}$ Benjamin P. McDermott ${ }^{\ddagger}$ and Hon Wai Lam* ${ }^{*} \dagger$ \\ ${ }^{\dagger}$ School of Chemistry, University of Edinburgh, Joseph Black Building, The King's Buildings, West \\ Mains Road, Edinburgh, EH9 3JJ, United Kingdom \\ ${ }^{\star}$ AstraZeneca R\&D Alderley Park, Macclesfield, Alderley Park, Macclesfield, Cheshire \\ SK10 $4 T F$
}

\section{Supporting Information}

\section{Contents}

General Information

Preparation of Alkynones

Preparation of Tethered Diacids

Double Michael Reactions

Preparation of 19-22

Stereochemical Determinations

NMR Spectra of New Compounds

$\mathrm{S} 13$

\section{General Information}

All non-aqueous reactions were carried out under a nitrogen atmosphere in oven-dried apparatus. $\mathrm{CH}_{2} \mathrm{Cl}_{2}$ and THF were dried and purified by passage through activated alumina columns using a solvent purification system from www.glasscontour.com 'Petrol' refers to that fraction of light petroleum ether boiling in the range $40-60{ }^{\circ} \mathrm{C}$. Thin layer chromatography (TLC) was performed on Merck DF-Alufoilien $60 \mathrm{~F}_{254} 0.2 \mathrm{~mm}$ precoated plates. Product spots were visualized by UV light at $254 \mathrm{~nm}$, and subsequently developed using potassium permanganate or ceric ammonium molybdate solution as appropriate. Flash column chromatography was carried out using silica gel (Fisher Scientific $60 \AA$ particle size 35-70 micron) employing the method of Still and co-workers. ${ }^{1}$ Melting points were recorded on a Gallenkamp melting point apparatus and are uncorrected. Infra-red spectra

1. Still, W. C.; Kahn, M.; Mitra, A. J. Org. Chem. 1978, 43, 2923-2925. 
were recorded on a Jasco FT/IR-460 Plus instrument as a thin film on sodium chloride plates or as a dilute solution in $\mathrm{CHCl}_{3}$. ${ }^{1} \mathrm{H}$ NMR spectra were recorded on a Bruker DPX360 (360 MHz) spectrometer or a Bruker ARX250 (250 MHz) spectrometer. Chemical shifts $(\delta)$ are quoted in parts per million (ppm) downfield of tetramethylsilane, using residual protonated solvent as internal standard $\left(\mathrm{CDCl}_{3}\right.$ at $\left.7.27 \mathrm{ppm}\right)$. Abbreviations used in the description of resonances are: $\mathrm{s}$ (singlet), $\mathrm{d}(\mathrm{doublet}), \mathrm{t}$ (triplet), q, (quartet), app (apparent), br (broad). Coupling constants $(J$ ) are quoted to the nearest 0.1 Hz. Proton-decoupled ${ }^{13} \mathrm{C}$ NMR spectra were recorded on a Bruker DPX360 (90.6 MHz) spectrometer or a Bruker ARX250 (62.9 MHz) spectrometer. Chemical shifts $(\delta)$ are quoted in parts per million (ppm) downfield of tetramethylsilane, using deuterated solvent as internal standard $\left(\mathrm{CDCl}_{3}\right.$ at 77.0 ppm). Assignments were made using the DEPT sequence with secondary pulses at $90^{\circ}$ and $135^{\circ}$. ${ }^{19} \mathrm{~F}$ NMR spectra were recorded on a Bruker ARX250 (235 MHz) spectrometer. Chemical shifts $(\delta)$ are quoted in parts per million (ppm) of $\mathrm{CFCl}_{3}$. High resolution mass spectra were recorded on a Finnigan MAT 900 XLT spectrometer using the electrospray (ES) positive ion mode at the EPSRC National Mass Spectrometry Service Centre, University of Wales Swansea, or on a Kratos MS50TC spectrometer using the fast atom bombardment (FAB) technique in the mass spectrometry laboratory at the School of Chemistry, University of Edinburgh. Stated calculated mass values refer to that of the ion (i.e. the actual species being detected), not that of the neutral parent compound.

\section{Preparation of Alkynones}

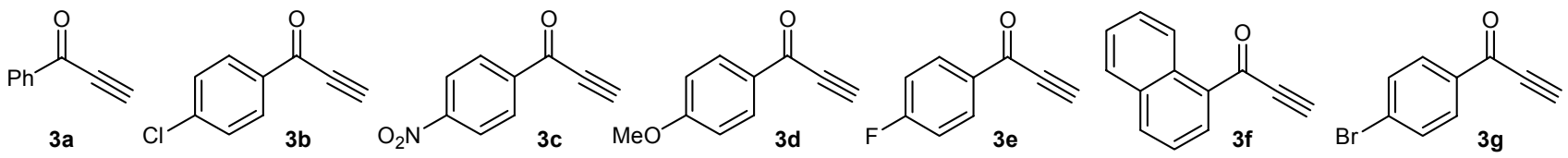

The known alkynones $\mathbf{3 a},{ }^{2} \mathbf{3 b},{ }^{3} \mathbf{3} \mathbf{c},{ }^{4} \mathbf{3 d},{ }^{5} \mathbf{3 e},{ }^{6} \mathbf{3} \mathbf{f},{ }^{2}$ and $\mathbf{3 \mathbf { g } ^ { 7 }}$ were all prepared by the addition of ethynyl magnesium bromide to $\mathrm{Ar}_{\mathrm{H}} \frac{1 . \equiv \mathrm{MgBr}_{2} \mathrm{Et}_{2} \mathrm{O}, 0^{\circ} \mathrm{C}}{2 \cdot \mathrm{MnO}_{2}, \mathrm{CH}_{2} \mathrm{Cl}_{2}, \mathrm{RT}}$ the appropriate aldehyde, followed by oxidation of the resulting propargylic alcohol with activated manganese dioxide.

2. $\quad$ Maeda, Y.; Kakiuchi, N.; Matsumura, S.; Nishimura, T.; Kawamura, T.; Uemura, S. J. Org. Chem. 2002, 67, 67186724.

3. $\quad$ Maeda, Y.; Washitake, Y.; Nishimura, T.; Iwai, K.; Yamauchi, T.; Uemura, S. Tetrahedron 2004, 60, 9031-9036.

4. $\quad$ Pigge, F. C.; Ghasedi, F.; Zheng, Z.; Rath, N. P.; Nichols, G.; Chickos, J. S. J. Chem. Soc., Perkin Trans 2 2000, 2458-2464.

5. $\quad$ Kumar, N.; Kiuchi, M.; Tallarico, J. A.; Schreiber, S. L. Org. Lett. 2005, 7, 2535-2538.

6. Bella, M.; Jørgensen, K. A. J. Am. Chem. Soc. 2004, 126, 5672-5673.

7. Alvarez-Ibarra, C.; Csáky, A. G.; Martín Ortega, E.; Jesús de la Morena, M.; Quiroga, M. L. Tetrahedron Lett. 1997, $38,4501-4052$. 


\section{Preparation of Tethered Diacids}

\section{Diethyl $N$-(2-carbethoxyacetyl)- $N$-(4-methoxyphenyl)aminomalonate (7a)}

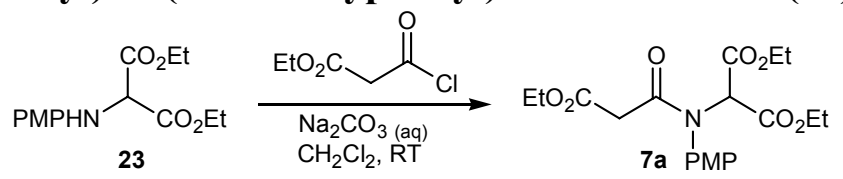

To a vigorously stirred mixture of the amine $23^{8}(1.00 \mathrm{~g}, 3.56 \mathrm{mmol})$ in $\mathrm{CH}_{2} \mathrm{Cl}_{2}(4 \mathrm{~mL})$ and saturated aqueous $\mathrm{Na}_{2} \mathrm{CO}_{3}$ solution $(4 \mathrm{~mL})$ at room temperature was added ethyl malonyl chloride $(0.61 \mathrm{~mL}$, $4.47 \mathrm{mmol}$ ) in one portion. The mixture was stirred for $1 \mathrm{~h}$ and then partitioned between saturated aqueous $\mathrm{NaHCO}_{3}$ solution $(30 \mathrm{~mL})$ and $\mathrm{CH}_{2} \mathrm{Cl}_{2}(30 \mathrm{~mL})$. The aqueous layer was separated and extracted with $\mathrm{CH}_{2} \mathrm{Cl}_{2}$ solution $(3 \times 50 \mathrm{~mL})$ and the combined organic layers were dried $\left(\mathrm{MgSO}_{4}\right)$ and concentrated in vacuo to leave the amide 7a (1.39 g, 98\%) as a brown oil. IR (film) 2983, 2938, 2840, $1742(\mathrm{C}=\mathrm{O}), 1672(\mathrm{C}=\mathrm{O}), 1510,1250,1034,843 \mathrm{~cm}^{-1} ;{ }^{1} \mathrm{H}$ NMR $\left(360 \mathrm{MHz}, \mathrm{CDCl}_{3}\right) \delta 7.41(2 \mathrm{H}, \mathrm{dm}, J$ $=8.9 \mathrm{~Hz}, \operatorname{ArH}), 6.88(2 \mathrm{H}, \mathrm{d}, J=8.9 \mathrm{~Hz}, \operatorname{ArH}), 5.55(1 \mathrm{H}, \mathrm{s}, \mathrm{CHN}), 4.23-4.09(6 \mathrm{H}, \mathrm{m}, 3 \mathrm{x}$ $\left.\mathrm{CO}_{2} \mathrm{CH}_{2} \mathrm{CH}_{3}\right), 3.82\left(3 \mathrm{H}, \mathrm{s}, \mathrm{OCH}_{3}\right), 3.24\left(2 \mathrm{H}, \mathrm{s}, \mathrm{CH}_{2} \mathrm{C}=\mathrm{O}\right), 1.29-1.14\left(9 \mathrm{H}, \mathrm{m}, 3 \times \mathrm{CO}_{2} \mathrm{CH}_{2} \mathrm{CH}_{3}\right) ;{ }^{13} \mathrm{C}$ NMR (62.9 MHz, $\left.\mathrm{CDCl}_{3}\right) \delta 166.9$ (2 x C), 165.6 (2 x C), 159.9 (C), 131.9 (C), 130.7 (2 x CH), 114.5 (2x CH), $64.3(\mathrm{CH}), 62.0\left(2 \times \mathrm{CH}_{2}\right), 61.2\left(\mathrm{CH}_{2}\right), 55.4\left(\mathrm{CH}_{3}\right), 41.4\left(\mathrm{CH}_{2}\right), 14.0\left(\mathrm{CH}_{3}\right), 13.8\left(2 \times \mathrm{CH}_{3}\right)$; HRMS (ES) Exact mass calcd for $\mathrm{C}_{19} \mathrm{H}_{26} \mathrm{NO}_{8}[\mathrm{M}+\mathrm{H}]^{+}: 395.1653$, found: 395.1654 .

\section{Dimethyl $N$-(2-carbethoxyacetyl)- $N$-(4-methoxyphenyl)aminomalonate (7b)}

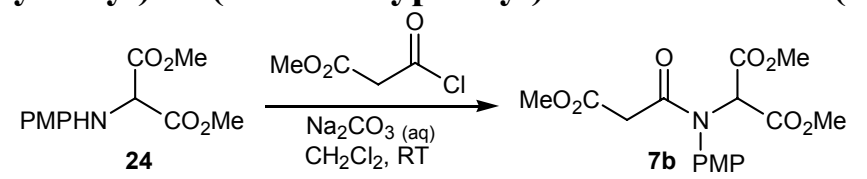

To a vigorously stirred mixture of the amine $24(3.00 \mathrm{~g}, 11.8 \mathrm{mmol})^{9}$ in $\mathrm{CH}_{2} \mathrm{Cl}_{2}(11 \mathrm{~mL})$ and saturated aqueous $\mathrm{Na}_{2} \mathrm{CO}_{3}$ solution $(11 \mathrm{~mL})$ at room temperature was added methyl malonyl chloride $(1.57 \mathrm{~mL}$, $14.2 \mathrm{mmol}$ ) over $1 \mathrm{~min}$. The mixture was stirred for $4 \mathrm{~h}$, and a further portion of methyl malonyl chloride $(0.65 \mathrm{mmol}, 5.92 \mathrm{mmol})$ was added. After stirring for another $1 \mathrm{~h}$, the reaction was partitioned between saturated aqueous $\mathrm{NaHCO}_{3}$ solution $(20 \mathrm{~mL})$ and $\mathrm{CH}_{2} \mathrm{Cl}_{2}(20 \mathrm{~mL})$. The aqueous layer was separated and extracted with $\mathrm{CH}_{2} \mathrm{Cl}_{2}$ solution $(3 \times 50 \mathrm{~mL})$ and the combined organic layers were dried $\left(\mathrm{MgSO}_{4}\right)$ and concentrated in vacuo to leave the amide $7 \mathbf{b}(4.00 \mathrm{~g}, 96 \%)$ as a brown oil. IR (film) 3004, 2954, 2843, $1747(\mathrm{C}=\mathrm{O}), 1671(\mathrm{C}=\mathrm{O}), 1509,1436,1249,1218,1167 ;{ }^{1} \mathrm{H} \mathrm{NMR}\left(360 \mathrm{MHz}, \mathrm{CDCl}_{3}\right) \delta$ $7.38(2 \mathrm{H}, \mathrm{dm}, J=8.3 \mathrm{~Hz}, \operatorname{ArH}), 6.89(2 \mathrm{H}, \mathrm{dm}, J=8.3 \mathrm{~Hz}, \operatorname{ArH}), 5.54(1 \mathrm{H}, \mathrm{s}, \mathrm{CHN}), 3.82(3 \mathrm{H}, \mathrm{s}$,

8. $\quad$ Simig, G.; Doleschall, G.; Hornyak, G.; Fetter, J.; Lempert, K.; Nyitrai, J.; Huszthy, P.; Gizur, T.; Kajtár-Peredy, M. Tetrahedron 1985, 41, 479-484.

9. Tombor, Z.; Greff, Z.; Nyitrai, J.; Kajtár-Peredy, M. Liebigs Ann. Org. Bioorg. Chem. 1995, 5, 825-836. 
$\left.\mathrm{OCH}_{3}\right), 3.72\left(6 \mathrm{H}, \mathrm{s}, 2\right.$ x $\left.\mathrm{OCH}_{3}\right), 3.69\left(3 \mathrm{H}, \mathrm{s}, \mathrm{OCH}_{3}\right), 3.26\left(2 \mathrm{H}, \mathrm{s}, \mathrm{CH}_{2} \mathrm{C}=\mathrm{O}\right) ;{ }^{13} \mathrm{C} \mathrm{NMR}(62.9 \mathrm{MHz}$, $\left.\mathrm{CDCl}_{3}\right) \delta 167.3(\mathrm{C}), 166.8(\mathrm{C}), 165.9(2 \times \mathrm{C}), 159.9(\mathrm{C}), 131.8(\mathrm{C}), 130.4(2 \times \mathrm{CH}), 114.6(2 \times \mathrm{CH})$, $64.1(\mathrm{CH}), 55.4\left(\mathrm{CH}_{3}\right), 52.8\left(2 \times \mathrm{CH}_{3}\right), 52.3\left(\mathrm{CH}_{3}\right), 41.1\left(\mathrm{CH}_{2}\right)$; HRMS (ES) Exact mass calcd for $\mathrm{C}_{16} \mathrm{H}_{20} \mathrm{NO}_{8}[\mathrm{M}+\mathrm{H}]^{+}:$353.1183, found: 353.1183 .

\section{Double Michael Reactions: General Procedure}

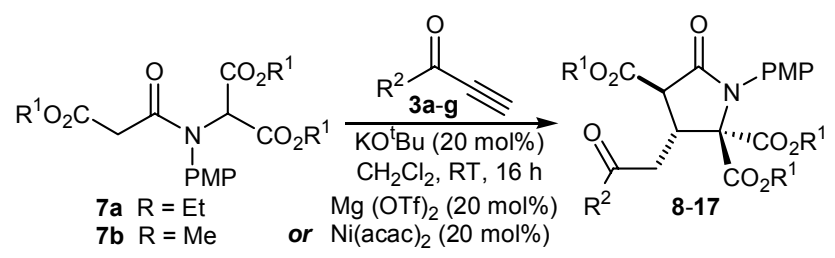

To a stirred solution of the appropriate tethered diacid $(0.20 \mathrm{mmol}), \mathrm{KO}^{\mathrm{t}} \mathrm{Bu}(4.7 \mathrm{mg}, 0.04 \mathrm{mmol})$ and the appropriate metal salt $(0.04 \mathrm{mmol})$ in $\mathrm{CH}_{2} \mathrm{Cl}_{2}(0.5 \mathrm{~mL})$ at room temperature was added a solution of the appropriate alkynone $(0.22 \mathrm{mmol})$ in $\mathrm{CH}_{2} \mathrm{Cl}_{2}(0.5 \mathrm{~mL})$ via cannula dropwise over $1 \mathrm{~min}$. The reaction was stirred at room temperature for $16 \mathrm{~h}$ and concentrated in vacuo. Purification of the residue by column chromatography afforded the double Michael product.

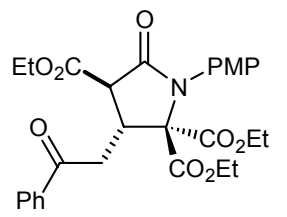

$( \pm)-(3 R, 4 R)-3,5,5-T r i c a r b e t h o x y-1-(4-m e t h o x y p h e n y l)-4-(2-0 x 0-2-$ phenylethyl)pyrrolidin-2-one (8). The title compound was prepared according to the General Procedure from tethered diacid $7 \mathbf{a}(79 \mathrm{mg}, 0.20 \mathrm{mmol})$, alkynone 3a (29 $\mathrm{mg}, 0.22 \mathrm{mmol})$ and $\mathrm{Ni}(\mathrm{acac})_{2}(10.8 \mathrm{mg}, 0.04 \mathrm{mmol})$ and purified by column chromatography $(20: 1$ $\left.\mathrm{CH}_{2} \mathrm{Cl}_{2} / \mathrm{Et}_{2} \mathrm{O}\right)$ to give a yellow oil $(80 \mathrm{mg}, 76 \%)$ as an 18:1 inseparable mixture of diastereomers. Trituration with $\mathrm{Et}_{2} \mathrm{O}$, followed by recrystallization from $\mathrm{Et}_{2} \mathrm{O} /$ hexane gave colorless crystals suitable for X-ray diffraction. m.p 101-102 ${ }^{\circ} \mathrm{C}$; IR (film) 3047, 2980, $1733(\mathrm{C}=\mathrm{O}), 1713(\mathrm{C}=\mathrm{O}), 1674(\mathrm{C}=\mathrm{O})$, 1509, 1249, 1053, 803, $778 \mathrm{~cm}^{-1} ;{ }^{1} \mathrm{H}$ NMR (360 MHz, $\left.\mathrm{CDCl}_{3}\right) \delta$ 7.97-7.95 (2H, m, ArH), 7.63-7.57 $(1 \mathrm{H}, \mathrm{m}, \operatorname{ArH}), 7.51-7.47(2 \mathrm{H}, \mathrm{m}, \operatorname{ArH}), 7.16(2 \mathrm{H}, \mathrm{d}, J=8.9 \mathrm{~Hz}, \operatorname{ArH}), 6.87(2 \mathrm{H}, \mathrm{d}, J=8.9 \mathrm{~Hz}, \operatorname{ArH})$, 4.37-4.24 (4H, m, $\left.2 \times \mathrm{OCH}_{2} \mathrm{CH}_{3}\right), 4.18-4.06\left(3 \mathrm{H}, \mathrm{m}, \mathrm{OCH}_{2} \mathrm{CH}_{3}\right.$ and $\left.\mathrm{CH}_{2} \mathrm{CH}\right), 3.80\left(3 \mathrm{H}, \mathrm{s}, \mathrm{OCH}_{3}\right)$, $3.64(1 \mathrm{H}, \mathrm{d}, J=11.3 \mathrm{~Hz}, \mathrm{CHC}=\mathrm{O}), 3.61\left(1 \mathrm{H}, \mathrm{dd}, J=16.7,4.7 \mathrm{~Hz}, \mathrm{CH}_{2} \mathrm{CH}\right), 3.02(1 \mathrm{H}, \mathrm{dd}, J=16.7,9.1$ $\left.\mathrm{Hz}, \mathrm{CH}_{2} \mathrm{CH}\right), 1.31-1.26\left(6 \mathrm{H}, \mathrm{m}, 2 \times \mathrm{OCH}_{2} \mathrm{CH}_{3}\right), 1.06\left(3 \mathrm{H}, \mathrm{t}, J=7.0 \mathrm{~Hz}, \mathrm{OCH}_{2} \mathrm{CH}_{3}\right) ;{ }^{13} \mathrm{C} \mathrm{NMR}(62.9$ $\left.\mathrm{MHz}, \mathrm{CDCl}_{3}\right) \delta 196.4(\mathrm{C}), 170.1$ (C), 168.4 (C), 167.9 (C), 166.1 (C), 159.2 (C), 136.1 (C), 133.5 (CH), $129.3(2 \times \mathrm{CH}), 129.1(\mathrm{C}), 128.7(2 \times \mathrm{CH}), 128.1(2 \times \mathrm{CH}), 114.0(2 \times \mathrm{CH}), 75.4(\mathrm{C}), 62.8$ $\left(\mathrm{CH}_{2}\right), 62.4\left(\mathrm{CH}_{2}\right), 61.9\left(\mathrm{CH}_{2}\right), 55.4\left(\mathrm{CH}_{3}\right), 52.7(\mathrm{CH}), 40.3(\mathrm{CH}), 39.1\left(\mathrm{CH}_{2}\right), 14.0\left(\mathrm{CH}_{3}\right), 14.0\left(\mathrm{CH}_{3}\right)$, 13.6 $\left(\mathrm{CH}_{3}\right)$; HRMS (ES) Exact mass calcd. for $\mathrm{C}_{28} \mathrm{H}_{32} \mathrm{NO}_{9}[\mathrm{M}+\mathrm{H}]^{+}:$526.2072, found: 526.2069. 


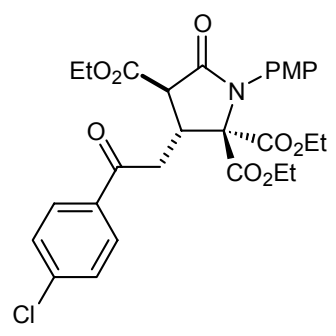

( \pm )-(3R,4R)-3,5,5-Tricarbethoxy-4-[2-(4-chlorophenyl)-2-oxoethyl]-1-(4methoxyphenyl)pyrrolidin-2-one (9). The title compound was prepared according to the General Procedure from tethered diacid $7 \mathbf{a}(79 \mathrm{mg}, 0.20 \mathrm{mmol})$, alkynone 3b (36 mg, $0.22 \mathrm{mmol})$ and $\mathrm{Mg}(\mathrm{OTf})_{2}(12.9 \mathrm{mg}, 0.04 \mathrm{mmol})$ and purified by column chromatography $\left(20: 1 \mathrm{CH}_{2} \mathrm{Cl}_{2} / \mathrm{Et}_{2} \mathrm{O}\right)$ to give a yellow oil (68 $\mathrm{mg}, 61 \%$ ) as a 14:1 inseparable mixture of diastereomers. IR (film) 2983, 2940, 2841, $1743(\mathrm{C}=\mathrm{O})$, $1673(\mathrm{C}=\mathrm{O}), 1510,1250,1035,844 \mathrm{~cm}^{-1} ;{ }^{1} \mathrm{H}$ NMR $\left(360 \mathrm{MHz}, \mathrm{CDCl}_{3}\right) \delta 7.91(2 \mathrm{H}, \mathrm{dm}, J=8.5 \mathrm{~Hz}$, $\operatorname{ArH}), 7.46(2 \mathrm{H}, \mathrm{dm}, J=8.5 \mathrm{~Hz}, \operatorname{ArH}), 7.15(2 \mathrm{H}, \mathrm{dm}, J=9.0 \mathrm{~Hz}, \operatorname{ArH}), 6.87(2 \mathrm{H}, \mathrm{dm}, J=9.0 \mathrm{~Hz}$, $\operatorname{ArH}), 4.42-4.25\left(4 \mathrm{H}, \mathrm{m}, 2\right.$ x $\left.\mathrm{OCH}_{2} \mathrm{CH}_{3}\right), 4.21-4.07\left(3 \mathrm{H}, \mathrm{m}, \mathrm{OCH}_{2} \mathrm{CH}_{3}\right.$ and $\left.\mathrm{CH}_{2} \mathrm{CH}\right), 3.79(3 \mathrm{H}, \mathrm{s}$, $\left.\mathrm{OCH}_{3}\right), 3.63(1 \mathrm{H}, \mathrm{d}, J=11.4 \mathrm{~Hz}, \mathrm{CHC}=\mathrm{O}), 3.58\left(1 \mathrm{H}, \mathrm{dd}, J=16.3,4.7 \mathrm{~Hz}, \mathrm{CH}_{2} \mathrm{CH}\right), 2.93(1 \mathrm{H}, \mathrm{dd}, J$ $\left.=16.4,9.2, \mathrm{CH}_{2} \mathrm{CH}\right), 1.29\left(3 \mathrm{H}, \mathrm{t}, J=7.1 \mathrm{~Hz}, \mathrm{OCH}_{2} \mathrm{CH}_{3}\right), 1.28\left(3 \mathrm{H}, \mathrm{t}, J=7.1 \mathrm{~Hz}, \mathrm{OCH}_{2} \mathrm{CH}_{3}\right), 1.05(3 \mathrm{H}$, $\left.\mathrm{t}, J=7.1 \mathrm{~Hz}, \mathrm{OCH}_{2} \mathrm{CH}_{3}\right) ;{ }^{13} \mathrm{C} \mathrm{NMR}\left(62.9 \mathrm{MHz} \mathrm{CDCl}_{3}\right) \delta 195.4$ (C), 170.0 (C), 168.4 (C), 167.9 (C), 166.1 (C), 159.2 (C), 140.0 (C), 134.4 (C), 129.5 (2 x CH), 129.3 (2 x CH), 129.0 (2 x CH and C), $114.1(2 \times \mathrm{CH}), 75.3(\mathrm{C}), 62.9\left(\mathrm{CH}_{2}\right), 62.5\left(\mathrm{CH}_{2}\right), 61.9\left(\mathrm{CH}_{2}\right), 55.4\left(\mathrm{CH}_{3}\right), 52.5(\mathrm{CH}), 40.3(\mathrm{CH}), 39.1$ $\left(\mathrm{CH}_{2}\right), 14.1\left(\mathrm{CH}_{3}\right), 14.0\left(\mathrm{CH}_{3}\right), 13.5\left(\mathrm{CH}_{3}\right)$; HRMS (ES) Exact mass calcd. for $\mathrm{C}_{28} \mathrm{H}_{31} \mathrm{ClNO}_{9}[\mathrm{M}+\mathrm{H}]^{+}$: 560.01682, found: 560.1683 .

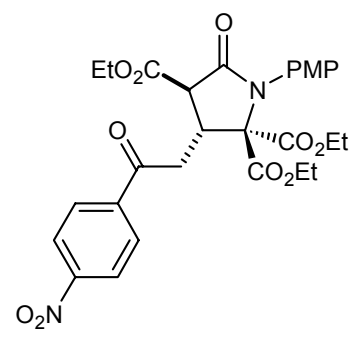

( \pm )-(3R,4R)-3,5,5-Tricarbethoxy-1-(4-methoxyphenyl)-4-[2-(4-nitrophenyl)2-oxoethyl]pyrrolidin-2-one (10). The title compound was prepared according to the General Procedure from tethered diacid $7 \mathbf{a}(79 \mathrm{mg}, 0.20 \mathrm{mmol})$, alkynone 3c $(39 \mathrm{mg}, 0.22 \mathrm{mmol})$ and $\mathrm{Ni}(\mathrm{acac})_{2}(10.8 \mathrm{mg}, 0.04 \mathrm{mmol})$ and purified by column chromatography (25\% EtOAc/petrol) to give a yellow oil (88 $\mathrm{mg}, 77 \%)$. IR (film) 2936, 2849, $1734(\mathrm{C}=\mathrm{O}), 1717(\mathrm{C}=\mathrm{O}), 1604,1526,1511,1348,1249,1223,1026 \mathrm{~cm}^{-1} ;{ }^{1} \mathrm{H}$ NMR $\left(360 \mathrm{MHz}, \mathrm{CDCl}_{3}\right) \delta 8.35(2 \mathrm{H}, \mathrm{dm}, J=8.9 \mathrm{~Hz}, \mathrm{ArH}), 8.15(2 \mathrm{H}, \mathrm{dm}, J=8.9 \mathrm{~Hz}, \operatorname{ArH}), 7.14(2 \mathrm{H}$, $\mathrm{dm}, J=9.0 \mathrm{~Hz}, \mathrm{ArH}), 6.88(2 \mathrm{H}, \mathrm{dm}, J=9.0 \mathrm{~Hz}, \mathrm{ArH}), 4.39-4.23$ (4H, m, 2 x $\left.\mathrm{OCH}_{2} \mathrm{CH}_{3}\right), 4.18-4.05$ $\left(3 \mathrm{H}, \mathrm{m}, \mathrm{OCH}_{2} \mathrm{CH}_{3}\right.$ and $\left.\mathrm{CH}_{2} \mathrm{CH}\right), 3.80\left(3 \mathrm{H}, \mathrm{s}, \mathrm{OCH}_{3}\right), 3.66(1 \mathrm{H}, \mathrm{dd}, J=16.3,4.6 \mathrm{~Hz}, \mathrm{CH} 2 \mathrm{CH}), 3.64$ $(1 \mathrm{H}, \mathrm{d}, J=11.4 \mathrm{~Hz}, \mathrm{CHC}=\mathrm{O}), 2.97\left(1 \mathrm{H}, \mathrm{dd}, J=16.3,9.2 \mathrm{~Hz}, \mathrm{CH}_{2} \mathrm{CH}\right), 1.31(3 \mathrm{H}, \mathrm{t}, J=7.1 \mathrm{~Hz}$, $\left.\mathrm{OCH}_{2} \mathrm{CH}_{3}\right), 1.30\left(3 \mathrm{H}, \mathrm{t}, J=7.2 \mathrm{~Hz}, \mathrm{OCH}_{2} \mathrm{CH}_{3}\right), 1.04\left(3 \mathrm{H}, \mathrm{t}, J=7.1 \mathrm{~Hz}, \mathrm{OCH}_{2} \mathrm{CH}_{3}\right) ;{ }^{13} \mathrm{C} \mathrm{NMR}(62.9$ $\mathrm{MHz}, \mathrm{CDCl}_{3}$ ) $\delta 195.4(\mathrm{C}), 169.6(\mathrm{C}), 168.2(\mathrm{C}), 167.8(\mathrm{C}), 166.0(\mathrm{C}), 159.3(\mathrm{C}), 150.5(\mathrm{C}), 140.3(\mathrm{C})$, $129.3(2 \times \mathrm{CH}), 129.2(2 \times \mathrm{CH}), 128.9(\mathrm{C}), 123.9(2 \times \mathrm{CH}), 114.1(2 \times \mathrm{CH}), 75.2(\mathrm{C}), 63.0\left(\mathrm{CH}_{2}\right), 62.6$ $\left(\mathrm{CH}_{2}\right), 62.0\left(\mathrm{CH}_{2}\right), 55.4\left(\mathrm{CH}_{3}\right), 52.2(\mathrm{CH}), 40.2(\mathrm{CH}), 39.6\left(\mathrm{CH}_{2}\right), 14.1\left(\mathrm{CH}_{3}\right), 14.0\left(\mathrm{CH}_{3}\right), 13.5\left(\mathrm{CH}_{3}\right)$; HRMS (ES) Exact mass calcd. for $\mathrm{C}_{28} \mathrm{H}_{31} \mathrm{~N}_{2} \mathrm{O}_{11}[\mathrm{M}+\mathrm{H}]^{+}$: 571.1922, found: 571.1923. 


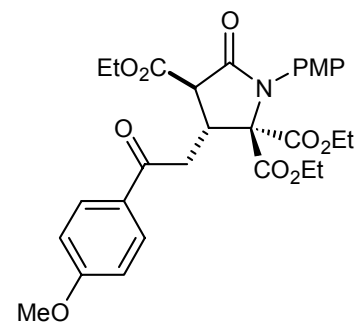

( \pm )-(3R,4R)-3,5,5-Tricarbethoxy-1-(4-methoxyphenyl)-4-[2-(4methoxyphenyl)-2-oxoethyl]pyrrolidin-2-one (11). The title compound was prepared according to the General Procedure from tethered diacid 7a $(79 \mathrm{mg}$, $0.20 \mathrm{mmol})$, alkynone $3 \mathbf{d}(35 \mathrm{mg}, 0.22 \mathrm{mmol})$ and $\mathrm{Mg}(\mathrm{OTf})_{2}(12.9 \mathrm{mg}, 0.04$ mmol) and purified by column chromatography (25\% EtOAc/petrol) to give a yellow oil (77 mg, 69\%) as a 10:1 inseparable mixture of diastereomers. IR (film) 2981, 2937, 2840, $1734(\mathrm{C}=\mathrm{O}), 1717(\mathrm{C}=\mathrm{O}), 1679(\mathrm{C}=\mathrm{O}), 1600,1511,1249,1171,1026 \mathrm{~cm}^{-1}$; ${ }^{1} \mathrm{H}$ NMR $(360 \mathrm{MHz}$, $\left.\mathrm{CDCl}_{3}\right) \delta 7.94(2 \mathrm{H}, \mathrm{dm}, J=9.0 \mathrm{~Hz}, \mathrm{ArH}), 7.15(2 \mathrm{H}, \mathrm{dm}, J=9.1 \mathrm{~Hz}, \mathrm{ArH}), 6.95(2 \mathrm{H}, \mathrm{dm}, J=9.0 \mathrm{~Hz}$, $\operatorname{ArH}), 6.87(2 \mathrm{H}, \mathrm{dm}, J=9.1 \mathrm{~Hz}, \mathrm{ArH}), 4.39-4.22\left(4 \mathrm{H}, \mathrm{m}, 2\right.$ x OCH $\left.\mathrm{CH}_{3}\right), 4.18-4.03\left(3 \mathrm{H}, \mathrm{m}, \mathrm{OCH}_{2} \mathrm{CH}_{3}\right.$ and $\left.\mathrm{CH}_{2} \mathrm{CH}\right), 3.88\left(3 \mathrm{H}, \mathrm{s}, \mathrm{OCH}_{3}\right), 3.80\left(3 \mathrm{H}, \mathrm{s}, \mathrm{OCH}_{3}\right), 3.64(1 \mathrm{H}, \mathrm{d}, J=11.3 \mathrm{~Hz}, \mathrm{CHC}=\mathrm{O}), 3.57(1 \mathrm{H}$, $\left.\mathrm{dd}, J=16.3,4.6 \mathrm{~Hz}, \mathrm{CH}_{2} \mathrm{CH}\right), 2.91\left(1 \mathrm{H}, \mathrm{dd}, J=16.3,9.3 \mathrm{~Hz}, \mathrm{CH}_{2} \mathrm{CH}\right), 1.28(3 \mathrm{H}, \mathrm{t}, J=7.1 \mathrm{~Hz}$, $\left.\mathrm{OCH}_{2} \mathrm{CH}_{3}\right), 1.27\left(3 \mathrm{H}, \mathrm{t}, J=7.1 \mathrm{~Hz}, \mathrm{OCH}_{2} \mathrm{CH}_{3}\right), 1.05\left(3 \mathrm{H}, \mathrm{t}, J=7.1 \mathrm{~Hz}, \mathrm{OCH}_{2} \mathrm{CH}_{3}\right) ;{ }^{13} \mathrm{C}$ NMR $(62.9$ $\left.\mathrm{MHz}, \mathrm{CDCl}_{3}\right) \delta 194.9(\mathrm{C}), 170.2$ (C), 168.4 (C), 167.9 (C), 166.1 (C), 163.7 (C), 159.2 (C), $130.4(2$ x $\mathrm{CH}), 129.3(2 \times \mathrm{CH}), 129.2(2 \times \mathrm{C}), 114.0(2 \times \mathrm{CH}), 113.8(2 \times \mathrm{CH}), 75.4(\mathrm{C}), 62.8\left(\mathrm{CH}_{2}\right), 62.4\left(\mathrm{CH}_{2}\right)$, $61.8\left(\mathrm{CH}_{2}\right), 55.5\left(\mathrm{CH}_{3}\right), 55.4\left(\mathrm{CH}_{3}\right), 52.7(\mathrm{CH}), 40.5(\mathrm{CH}), 38.7\left(\mathrm{CH}_{2}\right), 14.1\left(\mathrm{CH}_{3}\right), 14.0\left(\mathrm{CH}_{3}\right), 13.5$ $\left(\mathrm{CH}_{3}\right)$; HRMS (ES) Exact mass calcd. for $\mathrm{C}_{29} \mathrm{H}_{34} \mathrm{NO}_{10}[\mathrm{M}+\mathrm{H}]^{+}:$556.2177, found: 556.2175.

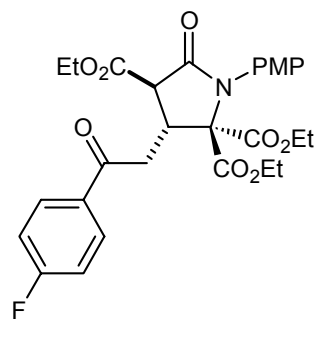

( \pm )-(3R,4R)-3,5,5-Tricarbethoxy-4-[2-(4-fluorophenyl)-2-oxoethyl]-1-(4methoxyphenyl)pyrrolidin-2-one (12). The title compound was prepared according to the General Procedure from tethered diacid $7 \mathbf{a}(79 \mathrm{mg}, 0.20 \mathrm{mmol})$, alkynone 3e (30 mg, $0.22 \mathrm{mmol})$ and $\mathrm{Mg}(\mathrm{OTf})_{2}(12.9 \mathrm{mg}, 0.04 \mathrm{mmol})$ and purified by column chromatography $\left(20: 1 \mathrm{CH}_{2} \mathrm{Cl}_{2} / \mathrm{Et}_{2} \mathrm{O}\right)$ to give a yellow oil (81 $\mathrm{mg}, 75 \%)$ as a 15:1 inseparable mixture of diastereomers. IR (film) 2982, 2937, 2840, $1735(\mathrm{C}=\mathrm{O})$, $1718(\mathrm{C}=\mathrm{O}), 1691(\mathrm{C}=\mathrm{O}), 1597,1511,1249,1230,1158 \mathrm{~cm}^{-1} ;{ }^{1} \mathrm{H}$ NMR $\left(360 \mathrm{MHz}, \mathrm{CDCl}_{3}\right) \delta 8.02-$ $7.98(2 \mathrm{H}, \mathrm{m}, \operatorname{ArH}), 7.17(2 \mathrm{H}, \mathrm{dm}, J=8.7 \mathrm{~Hz}, \operatorname{ArH}), 7.15(2 \mathrm{H}, \mathrm{dm}, J=9.0 \mathrm{~Hz}, \operatorname{ArH}), 6.87(2 \mathrm{H}, \mathrm{d}, J=$ $9.0 \mathrm{~Hz}, \mathrm{ArH}), 4.39-4.21\left(4 \mathrm{H}, \mathrm{m}, 2\right.$ x $\left.\mathrm{OCH}_{2} \mathrm{CH}_{3}\right), 4.17-4.03\left(3 \mathrm{H}, \mathrm{m}, \mathrm{OCH}_{2} \mathrm{CH}_{3}\right.$ and $\left.\mathrm{CH}_{2} \mathrm{CH}\right), 3.80(3 \mathrm{H}$, $\left.\mathrm{s}, \mathrm{OCH}_{3}\right), 3.63(2 \mathrm{H}, \mathrm{d}, J=11.4 \mathrm{~Hz}, \mathrm{CHC}=\mathrm{O}), 3.58\left(1 \mathrm{H}, \mathrm{dd}, J=16.4,4.6 \mathrm{~Hz}, \mathrm{CH}_{2} \mathrm{CH}\right), 2.93(1 \mathrm{H}, \mathrm{dd}, J$ $\left.=16.4,9.3 \mathrm{~Hz}, \mathrm{CH}_{2} \mathrm{CH}\right), 1.29\left(3 \mathrm{H}, \mathrm{t}, J=7.1 \mathrm{~Hz}, \mathrm{OCH}_{2} \mathrm{CH}_{3}\right), 1.28\left(3 \mathrm{H}, \mathrm{t}, J=7.2 \mathrm{~Hz}, \mathrm{OCH}_{2} \mathrm{CH}_{3}\right), 1.05$ $\left(3 \mathrm{H}, \mathrm{t}, J=7.2 \mathrm{~Hz}, \mathrm{OCH}_{2} \mathrm{CH}_{3}\right) ;{ }^{13} \mathrm{C} \mathrm{NMR}\left(62.9 \mathrm{MHz}, \mathrm{CDCl}_{3}\right) \delta 195.0(\mathrm{C}), 170.0(\mathrm{C}), 168.3(\mathrm{C}), 167.9$ (C), $166.0(\mathrm{C}), 165.9\left(\mathrm{C}, \mathrm{d}, J_{\mathrm{F}}=255.0 \mathrm{~Hz}\right), 159.2(\mathrm{C}), 132.5\left(\mathrm{C}, \mathrm{d}, J_{\mathrm{F}}=2.9 \mathrm{~Hz}\right), 130.8\left(2 \times \mathrm{CH}, \mathrm{d}, J_{\mathrm{F}}=\right.$ $9.4 \mathrm{~Hz}), 129.3(2 \times \mathrm{CH}), 129.0(\mathrm{C}), 115.8$ (2 x CH, d, J $=21.9 \mathrm{~Hz}), 114.0$ (2 x CH), 75.3 (C), 62.4 $\left(\mathrm{CH}_{2}\right), 62.8\left(\mathrm{CH}_{2}\right), 61.9\left(\mathrm{CH}_{2}\right), 55.3\left(\mathrm{CH}_{3}\right), 52.5(\mathrm{CH}), 40.3(\mathrm{CH}), 39.0\left(\mathrm{CH}_{2}\right), 14.0\left(\mathrm{CH}_{3}\right), 13.9\left(\mathrm{CH}_{3}\right)$, 
$13.5\left(\mathrm{CH}_{3}\right) ;{ }^{19} \mathrm{~F}$ NMR $\left(235 \mathrm{MHz}, \mathrm{CDCl}_{3}\right) \delta-104.6$; HRMS (ES) Exact mass calcd. for $\mathrm{C}_{28} \mathrm{H}_{31} \mathrm{FNO}_{9}$ $[\mathrm{M}+\mathrm{H}]^{+}:$544.1977, found: 544.1976.

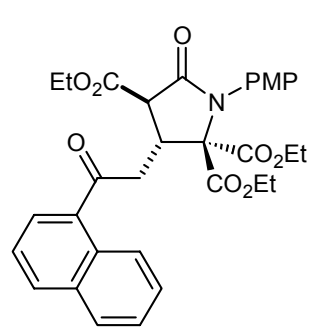

$( \pm)-(3 R, 4 R)-3,5,5-T r i c a r b e t h o x y-1-(4-m e t h o x y p h e n y l)-4-[2-(2-n a p h t h y l)-2-$ oxoethyl]pyrrolidin-2-one (13). The title compound was prepared according to the General Procedure from tethered diacid $7 \mathbf{a}(79 \mathrm{mg}, 0.20 \mathrm{mmol})$, alkynone $\mathbf{3 f}$ (40 mg, $0.22 \mathrm{mmol})$ and $\mathrm{Mg}(\mathrm{OTf})_{2}(12.9 \mathrm{mg}, 0.04 \mathrm{mmol})$ and purified by column chromatography $\left(20: 1 \mathrm{CH}_{2} \mathrm{Cl}_{2} / \mathrm{Et}_{2} \mathrm{O}\right)$ to give a yellow oil $(79 \mathrm{mg}, 69 \%)$ as an 8:1 inseparable mixture of diastereomers. IR (film) 2981, 2935, 2848, $1735(\mathrm{C}=\mathrm{O}), 1716(\mathrm{C}=\mathrm{O}), 1687$ $(\mathrm{C}=\mathrm{O}), 1511,1249,1214,1025 \mathrm{~cm}^{-1} ;{ }^{1} \mathrm{H}$ NMR $\left(360 \mathrm{MHz}, \mathrm{CDCl}_{3}\right) \delta$ 8.66-8.63 (1H, m, ArH), 8.048.02 (1H, m, ArH), 7.96-7.94 (1H, m, ArH), 7.90-7.88 (1H, m, ArH), 7.64-7.51 (3H, m, ArH), 7.18 $(2 \mathrm{H}, \mathrm{dm}, J=9.0 \mathrm{~Hz}, \mathrm{ArH}), 6.88(2 \mathrm{H}, \mathrm{dm}, J=9.0 \mathrm{~Hz}, \mathrm{ArH}), 4.40-4.09\left(7 \mathrm{H}, \mathrm{m}, 3 \mathrm{x} \mathrm{OCH}_{2} \mathrm{CH}_{3}\right.$ and $\left.\mathrm{CH}_{2} \mathrm{CH}\right), 3.80\left(3 \mathrm{H}, \mathrm{s}, \mathrm{OCH}_{3}\right), 3.68\left(1 \mathrm{H}, \mathrm{dd}, J=17.0,4.9 \mathrm{~Hz}, \mathrm{CH}_{2} \mathrm{CH}\right), 3.67(1 \mathrm{H}, \mathrm{d}, J=11.3 \mathrm{~Hz}$, $\mathrm{CHC}=\mathrm{O}), 3.16\left(1 \mathrm{H}, \mathrm{dd}, J=17.0,8.9 \mathrm{~Hz}, \mathrm{CH}_{2} \mathrm{CH}\right), 1.29\left(3 \mathrm{H}, \mathrm{t}, J=7.1 \mathrm{~Hz}, \mathrm{OCH}_{2} \mathrm{CH}_{3}\right), 1.25(3 \mathrm{H}, \mathrm{t}, J=$ $\left.7.2 \mathrm{~Hz}, \mathrm{OCH}_{2} \mathrm{CH}_{3}\right), 1.09\left(3 \mathrm{H}, \mathrm{t}, J=7.1 \mathrm{~Hz}, \mathrm{OCH}_{2} \mathrm{CH}_{3}\right) ;{ }^{13} \mathrm{C} \mathrm{NMR}\left(62.9 \mathrm{MHz}, \mathrm{CDCl}_{3}\right) \delta 200.0(\mathrm{C})$, 170.1 (C), 168.5 (C), 168.0 (C), 166.1 (C), 159.2 (C), 134.5 (C), 134.0 (C), 133.3 (CH), 130.1 (C), $129.2(2 \times \mathrm{CH}$ and $\mathrm{CH}), 128.4(\mathrm{CH}), 128.1(\mathrm{CH}), 128.0(\mathrm{CH}), 126.6(\mathrm{CH}), 125.8(\mathrm{CH}), 124.2(\mathrm{CH})$, $114.0(2 \times \mathrm{CH}), 73.4(\mathrm{C}), 62.8\left(\mathrm{CH}_{2}\right), 62.4\left(\mathrm{CH}_{2}\right), 61.9\left(\mathrm{CH}_{2}\right), 55.3\left(\mathrm{CH}_{3}\right), 52.9(\mathrm{CH}), 42.3\left(\mathrm{CH}_{2}\right), 40.7$ $(\mathrm{CH}), 14.0\left(2 \times \mathrm{CH}_{3}\right), 13.6\left(\mathrm{CH}_{3}\right)$; HRMS (ES) Exact mass calcd. for $\mathrm{C}_{32} \mathrm{H}_{34} \mathrm{NO}_{9}[\mathrm{M}+\mathrm{H}]^{+}$: 576.2228, found: 576.2231 .

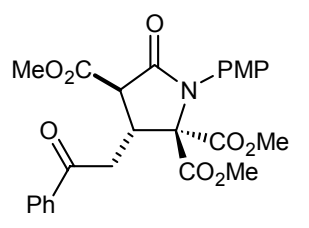

( \pm )-(3R,4R)-3,5,5-Tricarbomethoxy-1-(4-methoxyphenyl)-4-(2-oxo-2phenylethyl)pyrrolidin-2-one (14). The title compound was prepared according to the General Procedure from tethered diacid $7 \mathbf{b}(71 \mathrm{mg}, 0.20 \mathrm{mmol})$, alkynone $\mathbf{3 a}$ (29 $\mathrm{mg}, 0.22 \mathrm{mmol})$ and $\mathrm{Mg}(\mathrm{OTf})_{2}(12.9 \mathrm{mg}, 0.04 \mathrm{mmol})$ and purified by column chromatography $\left(20: 1 \mathrm{CH}_{2} \mathrm{Cl}_{2} / \mathrm{Et}_{2} \mathrm{O}\right)$ to give a yellow oil (58 $\left.\mathrm{mg}, 60 \%\right)$ as a 10:1 inseparable mixture of diastereomers. IR (film) 2954, $1740(\mathrm{C}=\mathrm{O}), 1716(\mathrm{C}=\mathrm{O}), 1687(\mathrm{C}=\mathrm{O}), 1511,1435,1248,830 \mathrm{~cm}^{-1} ;{ }^{1} \mathrm{H}$ NMR (360 MHz, $\left.\mathrm{CDCl}_{3}\right) \delta$ 7.96-7.93 (2H, m, ArH), 7.63-7.57 (1H, m, ArH), 7.51-7.47 (2H, m, ArH), $7.15(2 \mathrm{H}, \mathrm{dm}, J=8.9 \mathrm{~Hz}, \operatorname{ArH}), 6.89(2 \mathrm{H}, \mathrm{dm}, J=8.9 \mathrm{~Hz}, \operatorname{ArH}), 4.10(1 \mathrm{H}, \mathrm{ddd}, J=11.3,8.4,5.1 \mathrm{~Hz}$, $\left.\mathrm{CH}_{2} \mathrm{CH}\right), 3.67(1 \mathrm{H}, \mathrm{d}, J=11.3 \mathrm{~Hz}, \mathrm{CH}=\mathrm{O}), 3.83\left(3 \mathrm{H}, \mathrm{s}, \mathrm{OCH}_{3}\right), 3.81\left(3 \mathrm{H}, \mathrm{s}, \mathrm{OCH}_{3}\right), 3.80(3 \mathrm{H}, \mathrm{s}$, $\left.\mathrm{OCH}_{3}\right), 3.69\left(3 \mathrm{H}, \mathrm{s}, \mathrm{OCH}_{3}\right), 3.55\left(1 \mathrm{H}, \mathrm{dd}, J=17.2,5.1 \mathrm{~Hz}, \mathrm{CH}_{2} \mathrm{CH}\right), 3.07(1 \mathrm{H}, \mathrm{dd}, J=17.2,8.4 \mathrm{~Hz}$, $\left.\left.\mathrm{CH}_{2} \mathrm{CH}\right) ;{ }^{13} \mathrm{C} \mathrm{NMR}\left(62.9 \mathrm{MHz}, \mathrm{CDCl}_{3}\right) \delta 196.4(\mathrm{C}), 169.9(\mathrm{C}), 168.8(\mathrm{C}), 168 . \mathrm{x} \mathrm{CH}\right), 128.0(2 \times \mathrm{CH})$, 
$125.0(\mathrm{C}), 114.1(2 \times \mathrm{CH}), 75.6(\mathrm{C}), 55.3\left(\mathrm{CH}_{3}\right), 53.2\left(\mathrm{CH}_{3}\right), 53.1\left(\mathrm{CH}_{3}\right), 52.83(\mathrm{C}), 166.5(\mathrm{C}), 159.3$ (C), $136.0(\mathrm{C}), 133.5(\mathrm{CH}), 129.2(2 \times \mathrm{CH}), 128.8(\mathrm{C}), 128.7\left(2\left(\mathrm{CH}_{3}\right), 52.5(\mathrm{CH}), 40.4(\mathrm{CH}), 38.9\right.$ $\left(\mathrm{CH}_{2}\right)$; HRMS (ES) Exact mass calcd. for $\mathrm{C}_{25} \mathrm{H}_{26} \mathrm{NO}_{9}[\mathrm{M}+\mathrm{H}]^{+}$: 501.1868, found: 533.1875.

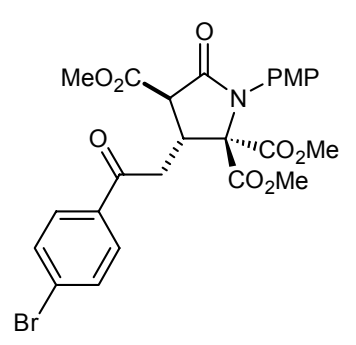

( \pm )-(3R,4R)-3,5,5-Tricarbomethoxy-4-[2-(4-bromophenyl)-2-oxoethyl]-1-(4methoxyphenyl)pyrrolidin-2-one (15). The title compound was prepared according to the General Procedure from tethered diacid $7 \mathbf{b}(71 \mathrm{mg}, 0.20 \mathrm{mmol})$, alkynone 3f (46 mg, $0.22 \mathrm{mmol})$ and $\operatorname{Mg}(\mathrm{OTf})_{2}(12.9 \mathrm{mg}, 0.04 \mathrm{mmol})$ and purified by column chromatography $\left(20: 1 \mathrm{CH}_{2} \mathrm{Cl}_{2} / \mathrm{Et}_{2} \mathrm{O}\right)$ to give a yellow oil (68 $\mathrm{mg}, 60 \%$ ) as a 10:1 inseparable mixture of diastereomers. IR (film) 2953, 2924, 2844, $1740(\mathrm{C}=\mathrm{O})$, $1716(\mathrm{C}=\mathrm{O}), 1690,1585,1511,1249 \mathrm{~cm}^{-1} ;{ }^{1} \mathrm{H}$ NMR $\left(360 \mathrm{MHz}, \mathrm{CDCl}_{3}\right) \delta 7.82(2 \mathrm{H}, \mathrm{d}, J=8.5 \mathrm{~Hz}$, $\operatorname{ArH}), 7.64(2 \mathrm{H}, \mathrm{d}, J=8.5 \mathrm{~Hz}, \operatorname{ArH}), 7.14(2 \mathrm{H}, \mathrm{dm}, J=9.0 \mathrm{~Hz}, \operatorname{ArH}), 6.88(2 \mathrm{H}, \mathrm{dm}, J=9.0 \mathrm{~Hz}$, ArH), $4.08\left(1 \mathrm{H}, \mathrm{ddd}, J=11.4,8.6,5.1 \mathrm{~Hz}, \mathrm{CH}_{2} \mathrm{CH}\right), 3.83\left(3 \mathrm{H}, \mathrm{s}, \mathrm{OCH}_{3}\right), 3.81\left(3 \mathrm{H}, \mathrm{s}, \mathrm{OCH}_{3}\right), 3.80$ $\left(3 \mathrm{H}, \mathrm{s}, \mathrm{OCH}_{3}\right), 3.68\left(3 \mathrm{H}, \mathrm{s}, \mathrm{OCH}_{3}\right), 3.67(1 \mathrm{H}, \mathrm{d}, J=11.4 \mathrm{~Hz}, \mathrm{CH}=\mathrm{O}), 3.51(1 \mathrm{H}, \mathrm{dd}, J=17.0,5.1 \mathrm{~Hz}$, $\left.\mathrm{CH}_{2} \mathrm{CH}\right), 3.00\left(1 \mathrm{H}, \mathrm{dd}, J=17.0,8.6 \mathrm{~Hz}, \mathrm{CH}_{2} \mathrm{CH}\right) ;{ }^{13} \mathrm{C} \mathrm{NMR}\left(62.9 \mathrm{MHz}, \mathrm{CDCl}_{3}\right) \delta 195.6(\mathrm{C}), 169.8$ (C), 168.8 (C), 168.3 (C), 166.6 (C), 159.4 (C), 134.7 (C), 132.1 (2 x CH), 129.6 (2 x CH), 129.3 (2 x $\mathrm{CH}), 128.9(\mathrm{C}), 128.8(\mathrm{C}), 114.2(2 \mathrm{x} \mathrm{CH}), 75.5(\mathrm{C}), 55.4\left(\mathrm{CH}_{3}\right), 53.3\left(\mathrm{CH}_{3}\right), 53.2\left(\mathrm{CH}_{3}\right), 53.0\left(\mathrm{CH}_{3}\right)$, $52.4(\mathrm{CH}), 40.4(\mathrm{CH}), 38.9\left(\mathrm{CH}_{2}\right)$; HRMS (ES) Exact mass calcd. for $\mathrm{C}_{25} \mathrm{H}_{25} \mathrm{BrNO}_{9}[\mathrm{M}+\mathrm{H}]^{+}$: 561.0707 , found: 561.0706 .

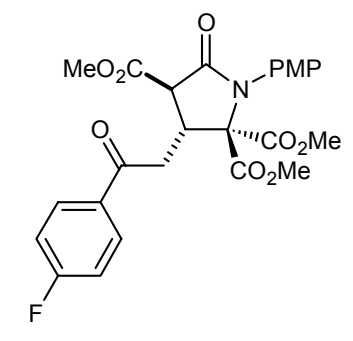

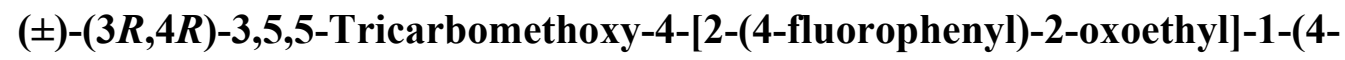
methoxyphenyl)pyrrolidin-2-one (16). The title compound was prepared according to the General Procedure from tethered diacid $7 \mathbf{b}(71 \mathrm{mg}, 0.20 \mathrm{mmol})$, alkynone $3 \mathbf{e}(30 \mathrm{mg}, 0.22 \mathrm{mmol})$ and $\mathrm{Ni}(\mathrm{acac})_{2}(10.8 \mathrm{mg}, 0.04 \mathrm{mmol})$ and purified by column chromatography $\left(20: 1 \mathrm{CH}_{2} \mathrm{Cl}_{2} / \mathrm{Et}_{2} \mathrm{O}\right)$ to give a yellow solid (59 $\mathrm{mg}$, $59 \%)$ as a 10:1 inseparable mixture of diastereomers. m.p. $146-147^{\circ} \mathrm{C}$; IR $\left(\mathrm{CHCl}_{3}\right) 2955,2924,2847$, $1741(\mathrm{C}=\mathrm{O}), 1716(\mathrm{C}=\mathrm{O}), 1689(\mathrm{C}=\mathrm{O}), 1597,1510,1249,1231,1158 \mathrm{~cm}^{-1} ;{ }^{1} \mathrm{H}$ NMR $(360 \mathrm{MHz}$, $\left.\mathrm{CDCl}_{3}\right) \delta$ 8.00-7.96 (2H, m, ArH), 7.18-7.13 (4H, m, $\left.\operatorname{ArH}\right), 6.88(2 \mathrm{H}, \mathrm{d}, J=9.0 \mathrm{~Hz}, \operatorname{ArH}), 4.08(1 \mathrm{H}$, ddd, $\left.J=11.3,8.6,5.1 \mathrm{~Hz}, \mathrm{CH}_{2} \mathrm{CH}\right), 3.82\left(3 \mathrm{H}, \mathrm{s}, \mathrm{OCH}_{3}\right), 3.81\left(3 \mathrm{H}, \mathrm{s}, \mathrm{OCH}_{3}\right), 3.80\left(3 \mathrm{H}, \mathrm{s}, \mathrm{OCH}_{3}\right), 3.67$ $(1 \mathrm{H}, \mathrm{d}, J=11.3 \mathrm{~Hz}, \mathrm{CHC}=\mathrm{O}), 3.52\left(1 \mathrm{H}, \mathrm{dd}, J=16.9,5.1 \mathrm{~Hz}, \mathbf{C H}_{2} \mathrm{CH}\right), 3.01(1 \mathrm{H}, \mathrm{dd}, J=16.9,8.6 \mathrm{~Hz}$, $\left.\mathrm{CH}_{2} \mathrm{CH}\right) ;{ }^{13} \mathrm{C}$ NMR $\left(62.9 \mathrm{MHz}, \mathrm{CDCl}_{3}\right) \delta 194.9(\mathrm{C}), 169.8(\mathrm{C}), 168.7$ (C), $168.2(\mathrm{C}), 166.6(\mathrm{C}), 165.9$ $\left(\mathrm{C}, \mathrm{d}, J_{\mathrm{F}}=255.5 \mathrm{~Hz}\right), 159.3(\mathrm{C}), 132.5\left(\mathrm{C}, \mathrm{d}, J_{\mathrm{F}}=2.8 \mathrm{~Hz}\right), 130.8\left(2 \mathrm{x} \mathrm{CH}, \mathrm{d}, J_{\mathrm{F}}=9.4 \mathrm{~Hz}\right), 129.2(2 \mathrm{x}$ 
$\mathrm{CH}), 128.8(\mathrm{C}), 115.8\left(2 \times \mathrm{CH}, \mathrm{d}, J_{\mathrm{F}}=22.0 \mathrm{~Hz}\right), 114.2(2 \times \mathrm{CH}), 75.5(\mathrm{C}), 55.3\left(\mathrm{CH}_{3}\right), 53.2\left(\mathrm{CH}_{3}\right)$, $53.1\left(\mathrm{CH}_{3}\right), 52.9\left(\mathrm{CH}_{3}\right), 52.4(\mathrm{CH}), 40.4(\mathrm{CH}), 38.8\left(\mathrm{CH}_{2}\right) ;{ }^{19} \mathrm{~F}$ NMR $\left(235 \mathrm{MHz}, \mathrm{CDCl}_{3}\right) \delta-104.4$; HRMS (ES) Exact mass calcd. for $\mathrm{C}_{25} \mathrm{H}_{25} \mathrm{FNO}_{9}[\mathrm{M}+\mathrm{H}]^{+}:$519.1773, found: 533.1777.

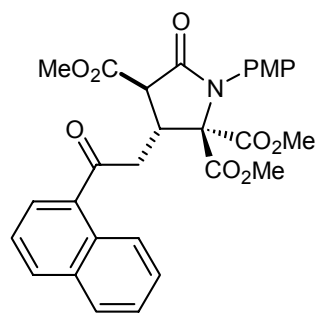

( \pm )-(3R,4R)-3,5,5-Tricarbomethoxy-1-(4-methoxyphenyl)-4-[2-(1-naphthyl)-2oxoethyl]pyrrolidin-2-one (17). The title compound was prepared according to the General Procedure from tethered diacid $7 \mathbf{b}(71 \mathrm{mg}, 0.20 \mathrm{mmol})$, alkynone $\mathbf{3 f}$ $(40 \mathrm{mg}, 0.22 \mathrm{mmol})$ and $\mathrm{Ni}(\mathrm{acac})_{2}(10.8 \mathrm{mg}, 0.04 \mathrm{mmol})$ and purified by column chromatography $\left(20: 1 \mathrm{CH}_{2} \mathrm{Cl}_{2} / \mathrm{Et}_{2} \mathrm{O}\right)$ to give a yellow oil (72 $\left.\mathrm{mg}, 67 \%\right)$ as a $15: 1$ inseparable mixture of diastereomers. IR (film) 2935, 2849, 2360, $1739(\mathrm{C}=\mathrm{O}), 1715(\mathrm{C}=\mathrm{O}), 1607$, 1510, 1435, 1364, $1250 \mathrm{~cm}^{-1} ;{ }^{1} \mathrm{H}$ NMR $\left(360 \mathrm{MHz}, \mathrm{CDCl}_{3}\right) \delta 8.61(1 \mathrm{H}, \mathrm{d}, J=7.8 \mathrm{~Hz}, \mathrm{ArH}), 8.04(1 \mathrm{H}$, d, $J=8.3 \mathrm{~Hz}, \operatorname{ArH}), 7.93-7.88(2 \mathrm{H}, \mathrm{m}, \operatorname{ArH}), 7.65-7.51(3 \mathrm{H}, \mathrm{m}, \operatorname{ArH}), 7.17(2 \mathrm{H}, \mathrm{d}, J=9.1 \mathrm{~Hz}, \operatorname{ArH})$, $6.89(2 \mathrm{H}, \mathrm{d}, J=9.1 \mathrm{~Hz}, \mathrm{ArH}), 4.21\left(1 \mathrm{H}, \mathrm{ddd}, J=11.3,8.3,5.6 \mathrm{~Hz}, \mathrm{CH}_{2} \mathrm{CH}\right), 3.84\left(3 \mathrm{H}, \mathrm{s}, \mathrm{OCH}_{3}\right), 3.81$ $\left(3 \mathrm{H}, \mathrm{s}, \mathrm{OCH}_{3}\right), 3.77\left(3 \mathrm{H}, \mathrm{s}, \mathrm{OCH}_{3}\right), 3.72\left(3 \mathrm{H}, \mathrm{s}, \mathrm{OCH}_{3}\right), 3.69(1 \mathrm{H}, \mathrm{d}, J=11.3 \mathrm{~Hz}, \mathrm{CHC}=\mathrm{O}), 3.59(1 \mathrm{H}$, $\left.\mathrm{dd}, J=17.4,5.6 \mathrm{~Hz}, \mathrm{CH}_{2} \mathrm{CH}\right), 3.23\left(1 \mathrm{H}, \mathrm{dd}, J=17.4,8.3 \mathrm{~Hz}, \mathrm{CH}_{2} \mathrm{CH}\right) ;{ }^{13} \mathrm{C} \mathrm{NMR}\left(62.9 \mathrm{MHz}, \mathrm{CDCl}_{3}\right)$ $\delta 200.0$ (C), 169.8 (C), 168.9 (C), 168.3 (C), 166.6 (C), 159.2 (C), 134.4 (C), 133.9 (C), $133.4(\mathrm{CH})$, $130.0(\mathrm{C}), 129.1(2 \times \mathrm{CH}), 128.8(\mathrm{C}), 128.4(\mathrm{CH}), 128.2(\mathrm{CH}), 127.9(\mathrm{CH}), 126.6(\mathrm{CH}), 125.7(\mathrm{CH})$, $124.2(\mathrm{CH}), 114.2(2 \times \mathrm{CH}), 75.6(\mathrm{C}), 55.3\left(\mathrm{CH}_{3}\right), 53.2\left(2 \times \mathrm{CH}_{3}\right), 52.9\left(\mathrm{CH}_{3}\right), 52.6(\mathrm{CH}), 42.2\left(\mathrm{CH}_{2}\right)$, $40.7(\mathrm{CH})$; HRMS (ES) Exact mass calcd. for $\mathrm{C}_{29} \mathrm{H}_{28} \mathrm{NO}_{9}[\mathrm{M}+\mathrm{H}]^{+}:$533.1759, found: 533.1759.

Diethyl $N$-(2-carbethoxy-5-oxohexanoyl)- $N$-(4-methoxyphenyl)aminomalonate (19) and diethyl 2[ $N$-(2-carbethoxyacetyl)- $N$-(4-methoxyphenyl)amino]-2-(3-oxobutyl)malonate (20)

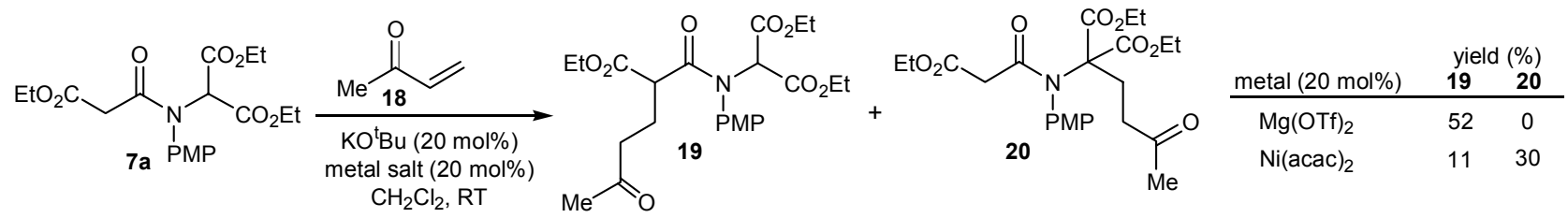

Using $\mathrm{Mg}(\mathrm{OTf})_{2}$ : To a stirred solution of the tethered diacid $7 \mathbf{a}(79 \mathrm{mg}, 0.20 \mathrm{mmol}), \mathrm{KO}^{\mathrm{t}} \mathrm{Bu}(4.7 \mathrm{mg}$, $0.04 \mathrm{mmol})$ and $\mathrm{Mg}(\mathrm{OTf})_{2}(12.9 \mathrm{mg}, 0.04 \mathrm{mmol})$ in $\mathrm{CH}_{2} \mathrm{Cl}_{2}(1 \mathrm{~mL})$ at room temperature was added methyl vinyl ketone $(20 \mu \mathrm{L}, 0.22 \mathrm{mmol})$. The reaction was stirred at room temperature for $16 \mathrm{~h}$ and concentrated in vacuo. Purification of the residue by column chromatography (25\% EtOAc/hexane $\rightarrow 50 \%$ EtOAc/hexane) gave the Michael adduct 19 (48 mg, 52\%) as a pale yellow oil. Using $\mathrm{Ni}\left(\mathrm{acac}_{2}\right.$ : To a stirred solution of the tethered diacid $7 \mathbf{a}(79 \mathrm{mg}, 0.20 \mathrm{mmol}), \mathrm{KO}^{\mathrm{t}} \mathrm{Bu}(4.7 \mathrm{mg}$, $0.04 \mathrm{mmol})$ and $\mathrm{Ni}(\mathrm{acac})_{2}(10.8 \mathrm{mg}, 0.04 \mathrm{mmol})$ in $\mathrm{CH}_{2} \mathrm{Cl}_{2}(1 \mathrm{~mL})$ at room temperature was added 
methyl vinyl ketone $(20 \mu \mathrm{L}, 0.22 \mathrm{mmol})$. The reaction was stirred at room temperature for $16 \mathrm{~h}$ and concentrated in vacuo. Purification of the residue by column chromatography (25\% EtOAc/hexane $\rightarrow 50 \%$ EtOAc/hexane) gave the Michael adduct 19 (10 mg, 11\%) as a pale yellow oil followed by the Michael adduct $\mathbf{2 0}(28 \mathrm{mg}, 30 \%)$ as a pale yellow oil.

Data for 19:

IR (film) 2983, 2938, 2908, $1741(\mathrm{C}=\mathrm{O}), 1716(\mathrm{C}=\mathrm{O}), 1668(\mathrm{C}=\mathrm{O}), 1509,1250,1180,1031 \mathrm{~cm}^{-1} ;{ }^{1} \mathrm{H}$ NMR $\left(360 \mathrm{MHz}, \mathrm{CDCl}_{3}\right) \delta 7.36(2 \mathrm{H}$, br s, ArH), $6.87(2 \mathrm{H}, \mathrm{d}, J=8.3 \mathrm{~Hz}, \mathrm{ArH}), 5.44(1 \mathrm{H}, \mathrm{s}, \mathrm{NCH})$, 4.28-4.00 (4H, m, 3 x $\left.\mathrm{OCH}_{2} \mathrm{CH}_{3}\right), 3.81\left(3 \mathrm{H}, \mathrm{s}, \mathrm{OCH}_{3}\right), 3.46\left(1 \mathrm{H}, \mathrm{dd}, J=8.1,6.3 \mathrm{~Hz}, \mathrm{CHCH}_{2}\right), 2.60-$ $2.43\left(2 \mathrm{H}, \mathrm{m}, \mathrm{CHCH}_{2} \mathrm{CH}_{2}\right), 2.12\left(3 \mathrm{H}, \mathrm{s}, \mathrm{CH}_{3} \mathrm{C}=\mathrm{O}\right), 2.10-2.05\left(2 \mathrm{H}, \mathrm{m}, \mathrm{CHCH}_{2}\right), 1.26(3 \mathrm{H}, \mathrm{t}, J=7.1 \mathrm{~Hz}$, $\left.\mathrm{OCH}_{2} \mathrm{CH}_{3}\right), 1.25\left(3 \mathrm{H}, \mathrm{t}, J=7.1 \mathrm{~Hz}, \mathrm{OCH}_{2} \mathrm{CH}_{3}\right), 1.16\left(3 \mathrm{H}, \mathrm{t}, J=7.1 \mathrm{~Hz}, \mathrm{OCH}_{2} \mathrm{CH}_{3}\right) ;{ }^{13} \mathrm{C}$ NMR $(62.9$ $\left.\mathrm{MHz}, \mathrm{CDCl}_{3}\right) \delta 207.7$ (C), 169.6 (C), 169.1 (C), 165.9 (C), 165.4 (C), 159.9 (C), 131.9 (C), 131.2 $(\mathrm{CH}), 130.3(\mathrm{CH}), 114.5(2 \times \mathrm{CH}), 64.9(\mathrm{CH}), 62.0\left(2 \times \mathrm{CH}_{2}\right), 61.3\left(\mathrm{CH}_{2}\right), 55.4\left(\mathrm{CH}_{3}\right), 47.6(\mathrm{CH}), 40.1$ $\left(\mathrm{CH}_{2}\right), 29.8\left(\mathrm{CH}_{3}\right), 23.1\left(\mathrm{CH}_{2}\right), 14.0\left(\mathrm{CH}_{3}\right), 13.9\left(\mathrm{CH}_{3}\right), 13.8\left(\mathrm{CH}_{3}\right)$; HRMS (ES) Exact mass calcd. for $\mathrm{C}_{23} \mathrm{H}_{32} \mathrm{NO}_{9}[\mathrm{M}+\mathrm{H}]^{+}:$466.2072, found: 466.2070 .

Data for 20: IR (film) 2982, 2939, 2841, $1741(\mathrm{C}=\mathrm{O}), 1671(\mathrm{C}=\mathrm{O}), 1509,1366,1248,1031,843 \mathrm{~cm}^{-1}$; ${ }^{1} \mathrm{H}$ NMR $\left(360 \mathrm{MHz}, \mathrm{CDCl}_{3}\right) \delta 7.36(2 \mathrm{H}, \mathrm{dm}, J=9.0 \mathrm{~Hz}, \mathrm{ArH}), 6.91(2 \mathrm{H}, \mathrm{dm}, J=9.0 \mathrm{~Hz}, \mathrm{ArH}), 4.19$ $\left(2 \mathrm{H}, \mathrm{q}, J=7.2 \mathrm{~Hz}, \mathrm{OCH}_{2} \mathrm{CH}_{3}\right), 4.19\left(2 \mathrm{H}, \mathrm{q}, J=7.1 \mathrm{~Hz}, \mathrm{OCH}_{2} \mathrm{CH}_{3}\right), 4.12(2 \mathrm{H}, \mathrm{q}, J=7.1 \mathrm{~Hz}$, $\left.\mathrm{OCH}_{2} \mathrm{CH}_{3}\right), 3.83\left(3 \mathrm{H}, \mathrm{s}, \mathrm{OCH}_{3}\right), 3.11\left(2 \mathrm{H}, \mathrm{s}, \mathrm{EtO}_{2} \mathrm{CCH}_{2}\right), 2.63-2.59\left(2 \mathrm{H}, \mathrm{m}, \mathrm{CH}_{2} \mathrm{CH} / \mathrm{C}=\mathrm{O}\right), 2.17-2.13$ $\left(2 \mathrm{H}, \mathrm{m}, \mathrm{CH}_{2} \mathrm{CH}_{2} \mathrm{C}=\mathrm{O}\right), 2.06\left(3 \mathrm{H}, \mathrm{s}, \mathrm{CH}_{3} \mathrm{C}=\mathrm{O}\right), 1.28\left(6 \mathrm{H}, \mathrm{t}, J=7.1 \mathrm{~Hz}, 2 \times \mathrm{OCH}_{2} \mathrm{CH}_{3}\right), 1.21(3 \mathrm{H}, \mathrm{t}, J=$ $\left.7.1 \mathrm{~Hz}, \mathrm{OCH}_{2} \mathrm{CH}_{3}\right) ;{ }^{13} \mathrm{C}$ NMR (62.9 MHz, $\left.\mathrm{CDCl}_{3}\right) \delta 206.6(\mathrm{C}), 167.6$ (2 x C), $167.4(\mathrm{C}), 167.0(\mathrm{C})$, $160.0(\mathrm{C}), 131.5(2 \times \mathrm{CH}), 131.4(\mathrm{C}), 114.6(2 \times \mathrm{CH}), 71.2(\mathrm{C}), 62.0\left(2 \times \mathrm{CH}_{2}\right), 61.1\left(\mathrm{CH}_{2}\right), 55.5\left(\mathrm{CH}_{3}\right)$, $42.4\left(\mathrm{CH}_{2}\right), 38.5\left(\mathrm{CH}_{2}\right), 29.9\left(\mathrm{CH}_{3}\right), 28.9\left(\mathrm{CH}_{2}\right), 14.0\left(2 \times \mathrm{CH}_{3}\right), 13.9\left(\mathrm{CH}_{3}\right)$; HRMS (ES) Exact mass calcd. for $\mathrm{C}_{23} \mathrm{H}_{32} \mathrm{NO}_{9}[\mathrm{M}+\mathrm{H}]^{+}$: 466.2072, found: 466.2072 .

$( \pm)-(3 R, 4 R)-4-B e n z y l$ 3,5,5-tricarbethoxy-1-(4-methoxyphenyl)-4-(2-oxo-2-phenylethyl)pyrrolidin2-one (21)

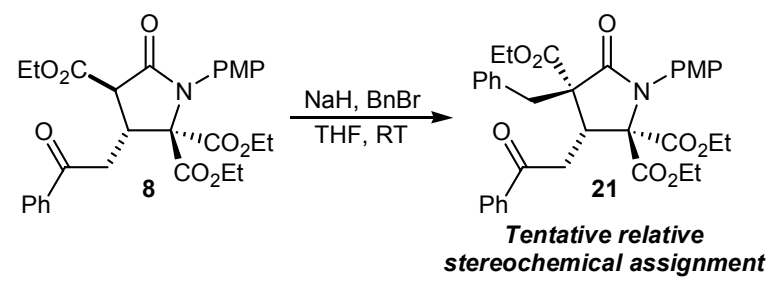

To a solution of the double Michael product $8(53 \mathrm{mg}, 0.10 \mathrm{mmol})$ in THF (1 mL) at room temperature was added $\mathrm{NaH}(60 \%$ dispersion in mineral oil, $40 \mathrm{mg}, 0.10 \mathrm{mmol})$ in one portion, followed by $\mathrm{BnBr}$ 
$(18 \mu \mathrm{L}, 0.15 \mathrm{mmol})$. The mixture was stirred at room temperature for $5 \mathrm{~h}$, quenched with $\mathrm{H}_{2} \mathrm{O}(10 \mathrm{~mL})$, and extracted with EtOAc $(3 \times 10 \mathrm{~mL})$, and the combined organic layers were dried $\left(\mathrm{MgSO}_{4}\right)$ and concentrated in vacuo. Purification of the residue by column chromatography (20\% EtOAc/hexane $\rightarrow 33 \%$ EtOAc/hexane) gave the benzylated pyrrolidinone 21 (41 mg, 67\%) as a yellow oil. IR (film) 2982, 2936, 2904, $1741(\mathrm{C}=\mathrm{O}), 1706(\mathrm{C}=\mathrm{O}), 1510,1249,1070,1030,913 \mathrm{~cm}^{-1}$; ${ }^{1} \mathrm{H}$ NMR $\left(360 \mathrm{MHz}, \mathrm{CDCl}_{3}\right) \delta$ 7.94-7.91 $(2 \mathrm{H}, \mathrm{m}, \mathrm{ArH}), 7.59(1 \mathrm{H}, \mathrm{t}, J=7.4 \mathrm{~Hz}, \mathrm{ArH}), 7.48(2 \mathrm{H}$, app t, $J=7.6$ Hz, ArH), 7.32 (5H, br s, ArH), 7.19 (2H, dm, $J=8.9 \mathrm{~Hz}, \operatorname{ArH}), 6.83$ (2H, dm, J=8.8 Hz, ArH), 4.31$4.25\left(2 \mathrm{H}, \mathrm{m}, \mathrm{OCH}_{2} \mathrm{CH}_{3}\right), 4.15-3.91\left(5 \mathrm{H}, \mathrm{m}, 2 \times \mathrm{OCH}_{2} \mathrm{CH}_{3}\right.$ and $\left.\mathrm{CH}_{2} \mathrm{CH}\right), 3.79(3 \mathrm{H}, \mathrm{s}, \mathrm{OCH} 3), 3.64$ $\left(1 \mathrm{H}, \mathrm{dd}, J=18.2,10.8 \mathrm{~Hz}, \mathrm{CH}_{2} \mathrm{CH}\right), 3.64\left(1 \mathrm{H}, \mathrm{d}, J=14.1 \mathrm{~Hz}, \mathrm{CH}_{2} \mathrm{Ph}\right), 3.24(1 \mathrm{H}, \mathrm{d}, J=14.1 \mathrm{~Hz}$, $\left.\mathrm{CH}_{2} \mathrm{Ph}\right), 3.10\left(1 \mathrm{H}, \mathrm{dd}, J=18.2,4.0 \mathrm{~Hz}, \mathrm{CH}_{2} \mathrm{CH}\right), 1.35\left(3 \mathrm{H}, \mathrm{t}, J=7.2 \mathrm{~Hz}, \mathrm{OCH}_{2} \mathrm{CH}_{3}\right), 1.00-0.95(6 \mathrm{H}$, m, $\left.2 \times \mathrm{OCH}_{2} \mathrm{CH}_{3}\right) ;{ }^{13} \mathrm{C} \mathrm{NMR}\left(62.9 \mathrm{MHz}, \mathrm{CDCl}_{3}\right) \delta 196.4(\mathrm{C}), 172.0(\mathrm{C}), 169.9(\mathrm{C}), 167.4(\mathrm{C}), 167.1$ (C), $159.1(\mathrm{C}), 136.4(\mathrm{C}), 135.4(\mathrm{C}), 133.2(\mathrm{CH}), 131.0(2 \times \mathrm{CH}), 130.4(2 \times \mathrm{CH}), 129.3(\mathrm{C}), 128.6(2 \times$ $\mathrm{CH}), 128.4(2 \times \mathrm{CH}), 127.9(2 \times \mathrm{CH}), 127.3(\mathrm{CH}), 113.7(2 \times \mathrm{CH}), 75.7(\mathrm{C}), 62.3\left(\mathrm{CH}_{2}\right), 61.9\left(\mathrm{CH}_{2}\right)$, $61.7\left(\mathrm{CH}_{2}\right), 58.6(\mathrm{C}), 55.3\left(\mathrm{CH}_{3}\right), 40.4(\mathrm{CH}), 37.4\left(\mathrm{CH}_{2}\right), 36.7\left(\mathrm{CH}_{2}\right), 14.1\left(\mathrm{CH}_{3}\right), 13.6\left(2 \times \mathrm{CH}_{3}\right)$; HRMS (ES) Exact mass calcd. for $\mathrm{C}_{35} \mathrm{H}_{38} \mathrm{NO}_{9}[\mathrm{M}+\mathrm{H}]^{+}:$616.2541, found: 616.2538.

\section{( \pm )-(4R)-5,5-Dicarbethoxy-1-(4-methoxyphenyl)-4-(2-oxo-2-phenylethyl)pyrrolidin-2-one (22)}

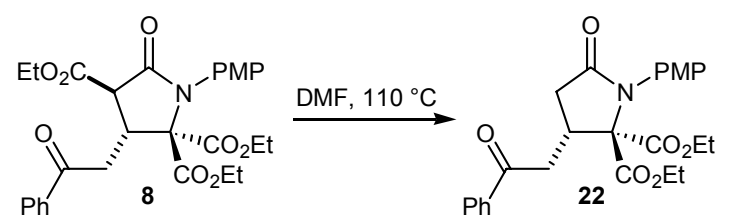

A solution of double Michael product $8(53 \mathrm{mg}, 0.10 \mathrm{mmol})$ in DMF $(1 \mathrm{~mL})$ was heated to $110{ }^{\circ} \mathrm{C}$ for $72 \mathrm{~h}$. After cooling to room temperature, the mixture was partitioned between $\mathrm{H}_{2} \mathrm{O}(15 \mathrm{~mL})$ and EtOAc $(15 \mathrm{~mL})$. The aqueous layer was separated and extracted with EtOAc $(2 \times 15 \mathrm{~mL})$, and the combined organic layers were dried $\left(\mathrm{MgSO}_{4}\right)$ and concentrated in vacuo. Purification of the residue by column chromatography (25\% EtOAc/hexane $\rightarrow 33 \%$ EtOAc/hexane) gave the decarboxylated pyrrolidinone 22 (27 mg, 60\%) as a yellow oil. IR (film) 2981, 2936, 2838, 1730 (C=O), 1687 (C=O), 1511, 1294, 1248, 1031, $690 \mathrm{~cm}^{-1} ;{ }^{1} \mathrm{H}$ NMR (360 MHz, $\left.\mathrm{CDCl}_{3}\right) \delta 7.96(2 \mathrm{H}, \mathrm{d}, J=7.3 \mathrm{~Hz}, \operatorname{ArH}), 7.63-7.59$ (1H, m, ArH), 7.52-7.47 (2H, m, ArH), 7.17 (2H, dm, $J=8.9 \mathrm{~Hz}, \operatorname{ArH}), 6.88(2 \mathrm{H}, \mathrm{dm}, J=8.9 \mathrm{~Hz}, \operatorname{ArH}), 4.40-4.26$ $\left(2 \mathrm{H}, \mathrm{m}, \mathrm{OCH}_{2} \mathrm{CH}_{3}\right), 4.19-4.03\left(2 \mathrm{H}, \mathrm{m}, \mathrm{OCH}_{2} \mathrm{CH}_{3}\right), 3.80(3 \mathrm{H}, \mathrm{s}, \mathrm{OCH})_{3}, 3.80-3.70\left(1 \mathrm{H}, \mathrm{m}, \mathrm{CH}_{2} \mathrm{CH}\right)$, $3.54\left(1 \mathrm{H}, \mathrm{dd}, J=17.2,3.9 \mathrm{~Hz}, \mathrm{PhCOCH}_{2}\right), 3.02-2.94\left(2 \mathrm{H}, \mathrm{m}, \mathrm{CH}_{2} \mathrm{CON}\right.$ and $\left.\mathrm{PhCOCH}_{2}\right), 2.40(1 \mathrm{H}, \mathrm{dd}$, $\left.J=16.7,11.1 \mathrm{~Hz}, \mathrm{CH}_{2} \mathrm{CON}\right), 1.30$ (3H, t, $J=7174.8$ (C), 168.1 (C), 166.8 (C), 159.1 (C), 136.3 (C), $133.5(\mathrm{CH}), 129.5(\mathrm{C}$ and $\left.2 \times \mathrm{CH}), 128.8(2 \times \mathrm{CH}), 128.1 \mathrm{~Hz}, \mathrm{OCH}_{2} \mathrm{CH}_{3}\right), 1.06(3 \mathrm{H}, \mathrm{t}, J=7.1 \mathrm{~Hz}$, 
$\left.\mathrm{OCH}_{2} \mathrm{CH}_{3}\right) ;{ }^{13} \mathrm{C} \mathrm{NMR}\left(62.9 \mathrm{MHz}, \mathrm{CDCl}_{3}\right) \delta 196.7(\mathrm{C}), .0(2 \times \mathrm{CH}), 114.1(2 \times \mathrm{CH}), 62.5\left(\mathrm{CH}_{2}\right), 62.3$ $\left(\mathrm{CH}_{2}\right), 60.4(\mathrm{C}), 55.4\left(\mathrm{CH}_{3}\right), 39.8\left(\mathrm{CH}_{2}\right), 36.7(\mathrm{CH}), 35.6\left(\mathrm{CH}_{2}\right), 14.2\left(\mathrm{CH}_{3}\right), 13.6\left(\mathrm{CH}_{3}\right)$; HRMS (ES) Exact mass calcd. for $\mathrm{C}_{25} \mathrm{H}_{28} \mathrm{NO}_{7}[\mathrm{M}+\mathrm{H}]^{+}: 454.1860$, found: 454.1863 .

\section{Stereochemical Determinations}

- The relative stereochemistry of $\mathbf{8}$ was determined by X-ray crystallography. Crystal structure deposited at the Cambridge Crystallographic Data Center; Deposition Number: CCDC 638217 Note - there is a small degree of disorder associated with one of the geminal diester groups (see CIF file for details).
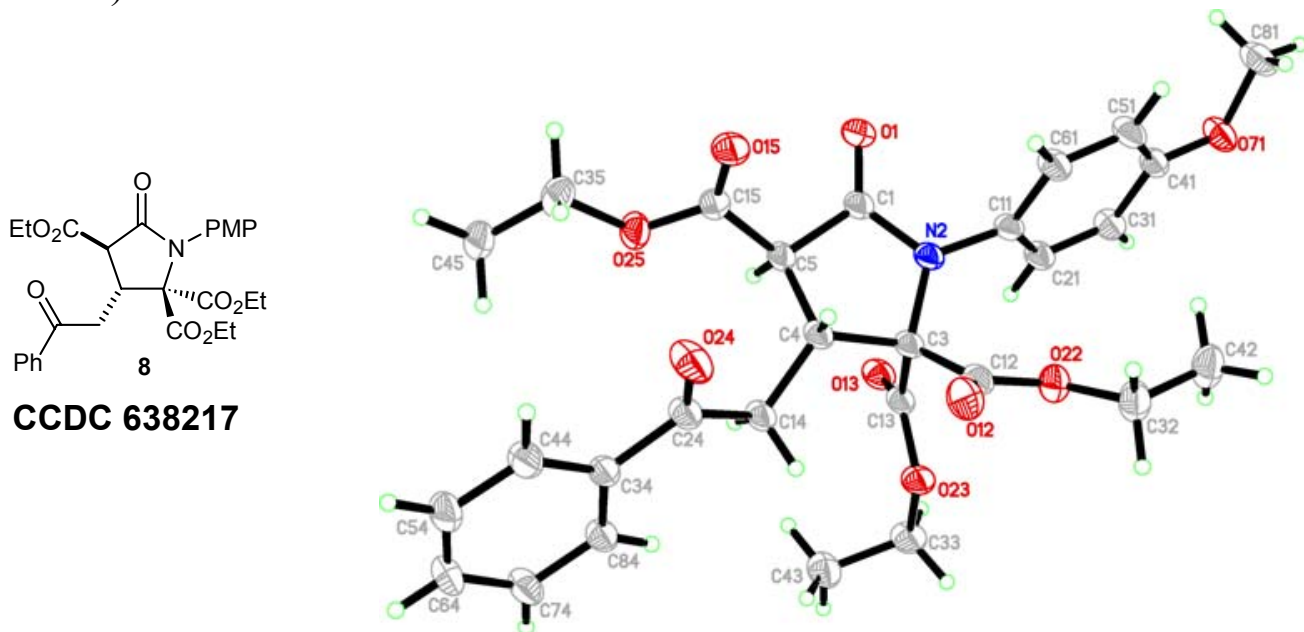

- The relative stereochemistries of the remaining double Michael products 9-17 were assigned by analogy. 


\section{NMR Spectra of New Compounds}
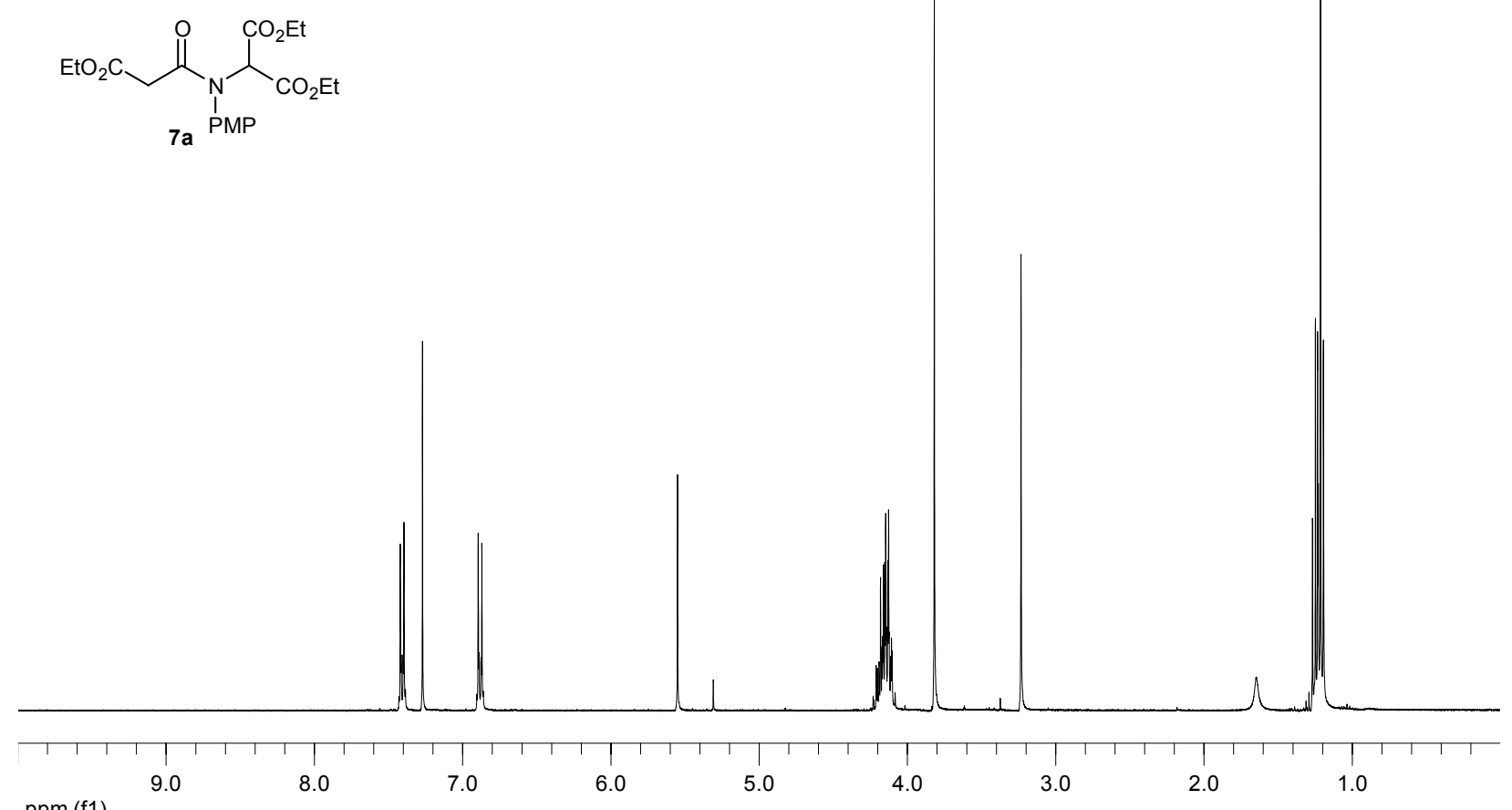

ppm (f1)
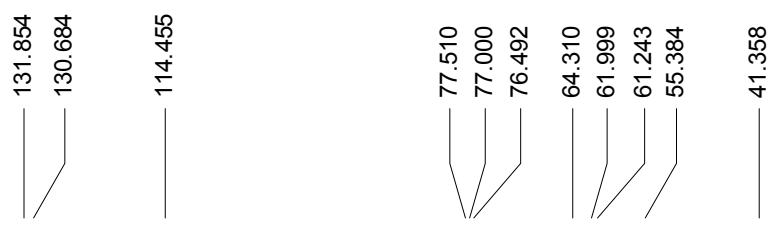

గ

응 苋术

@

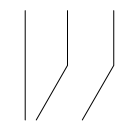

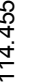
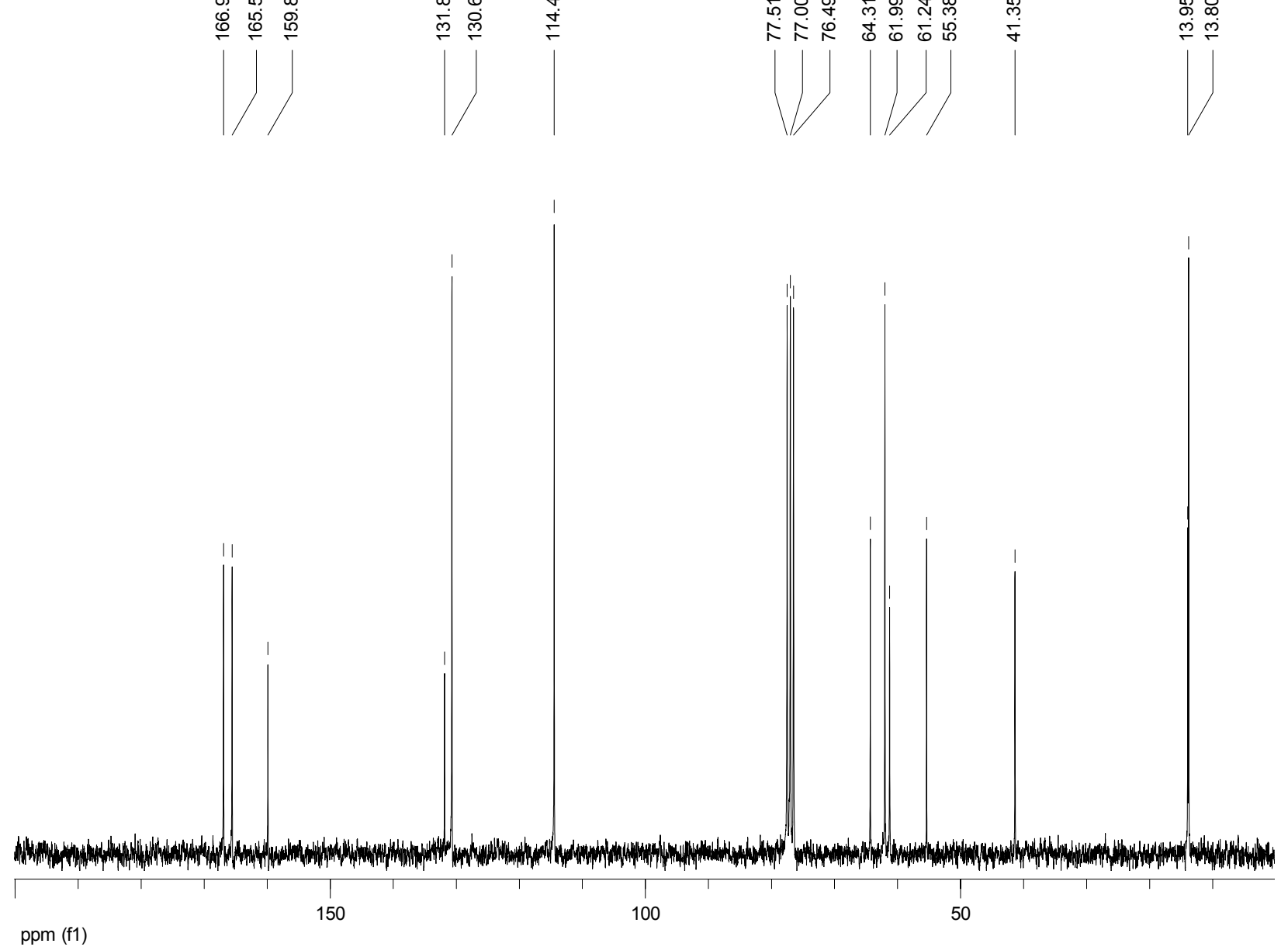

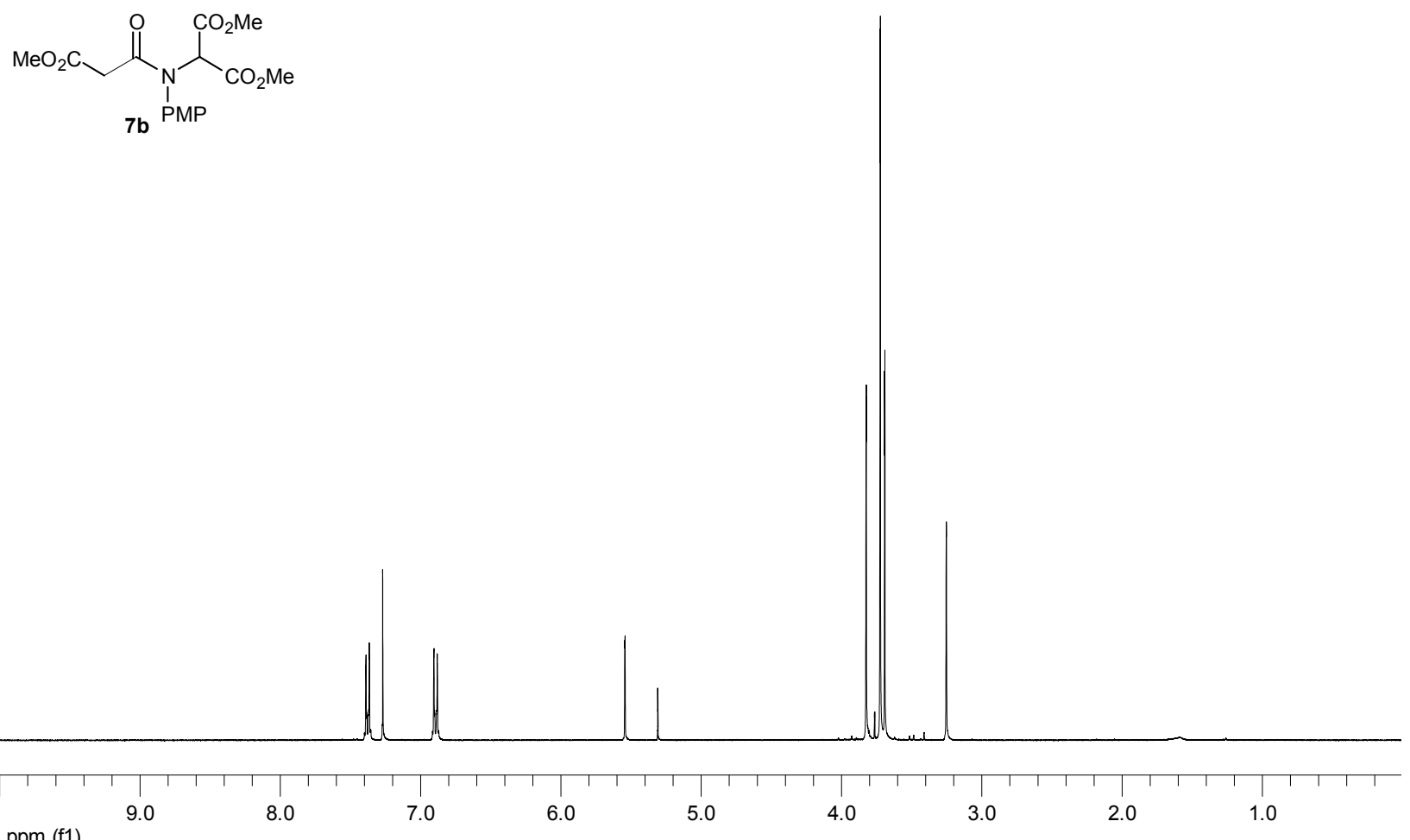

ppm (f1)

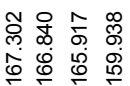

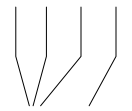

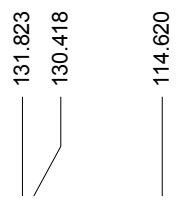
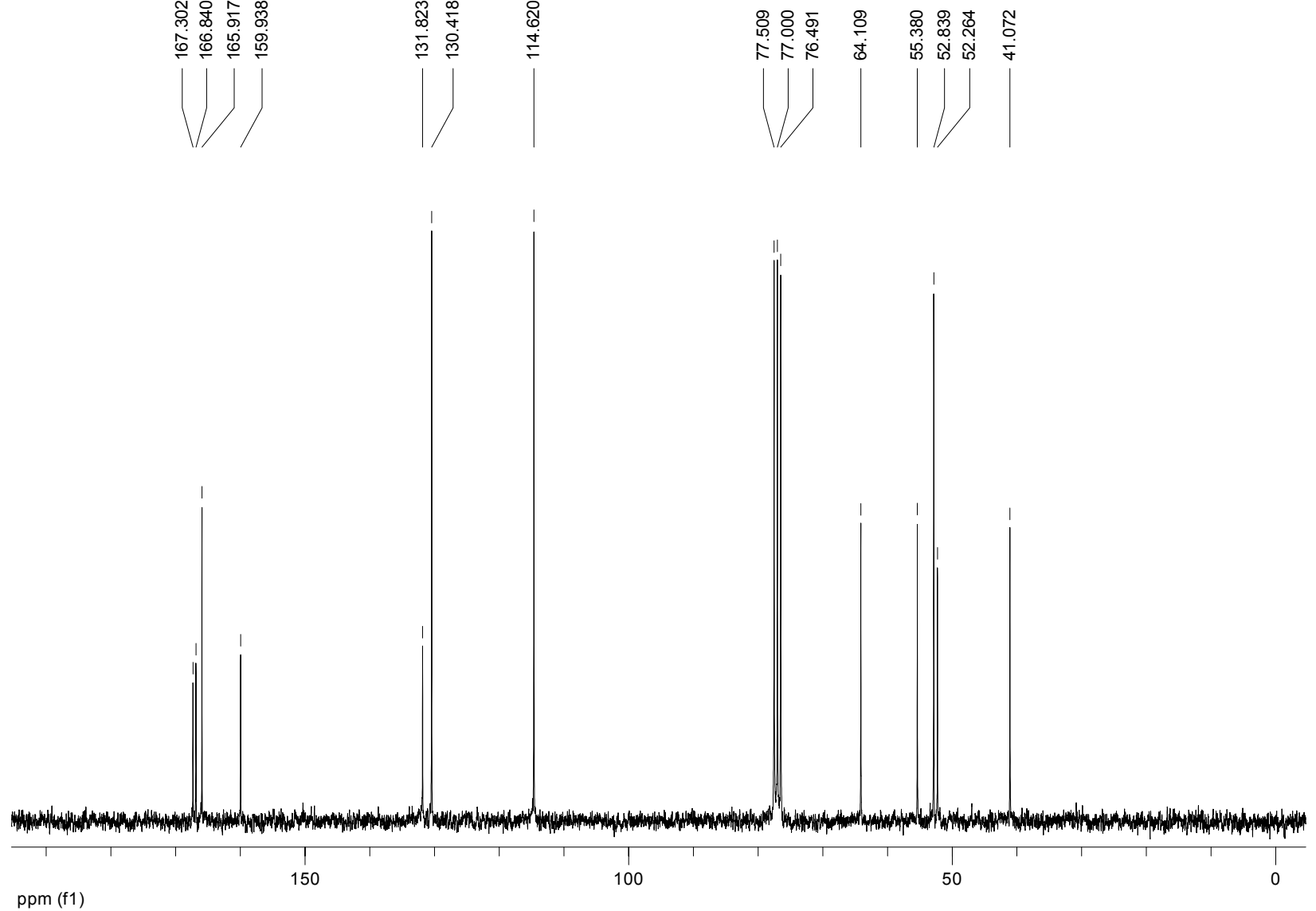

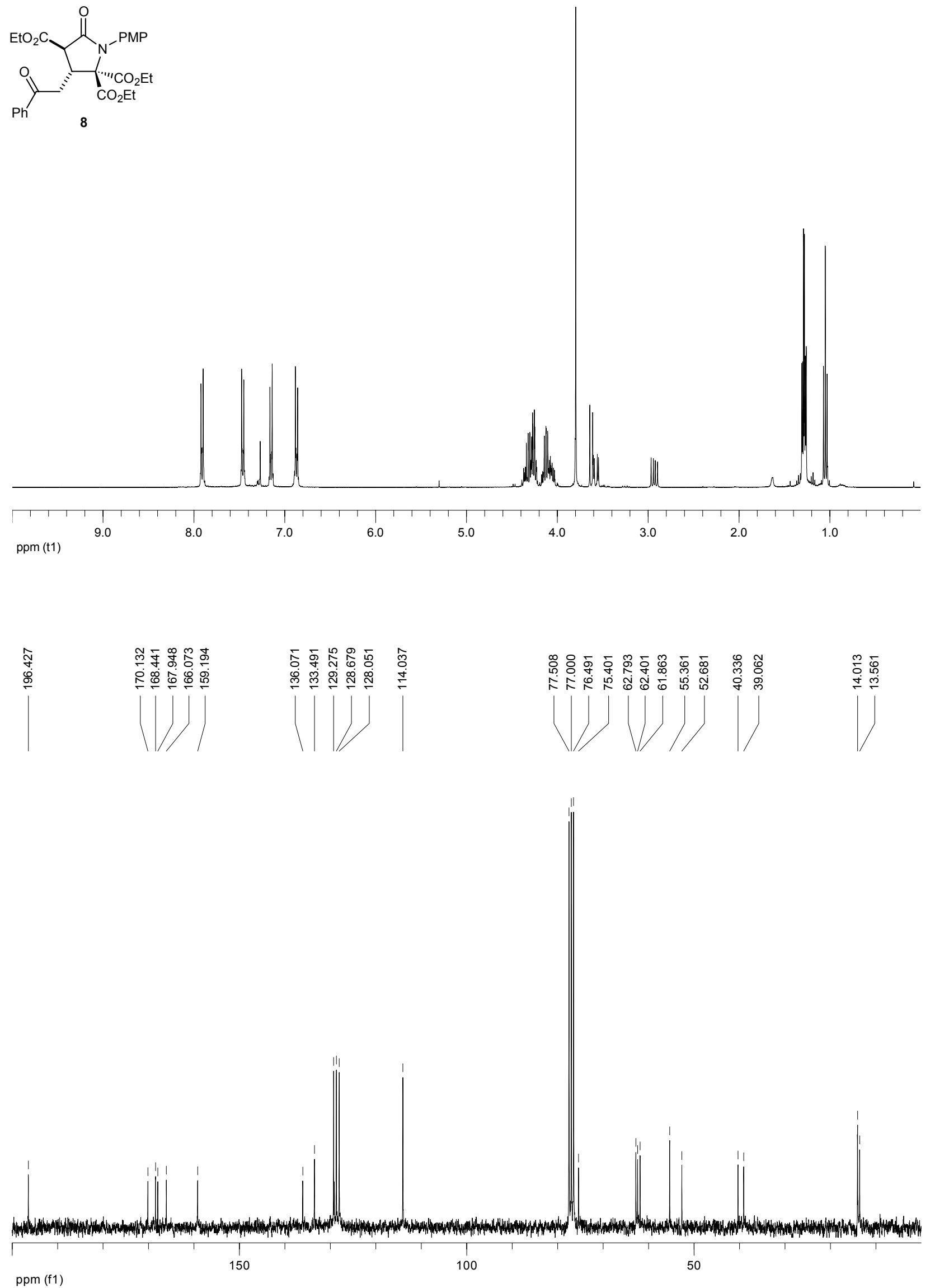

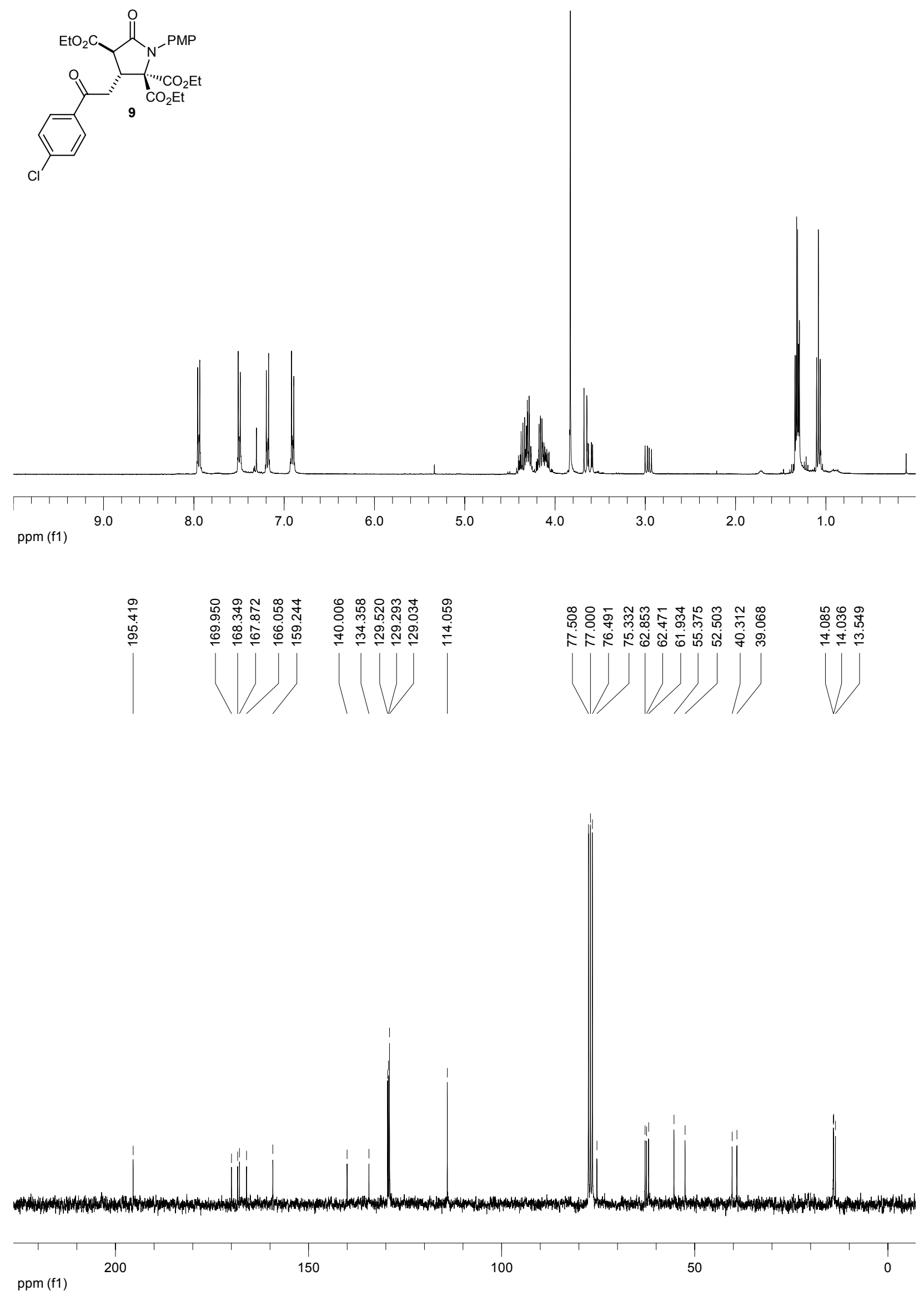

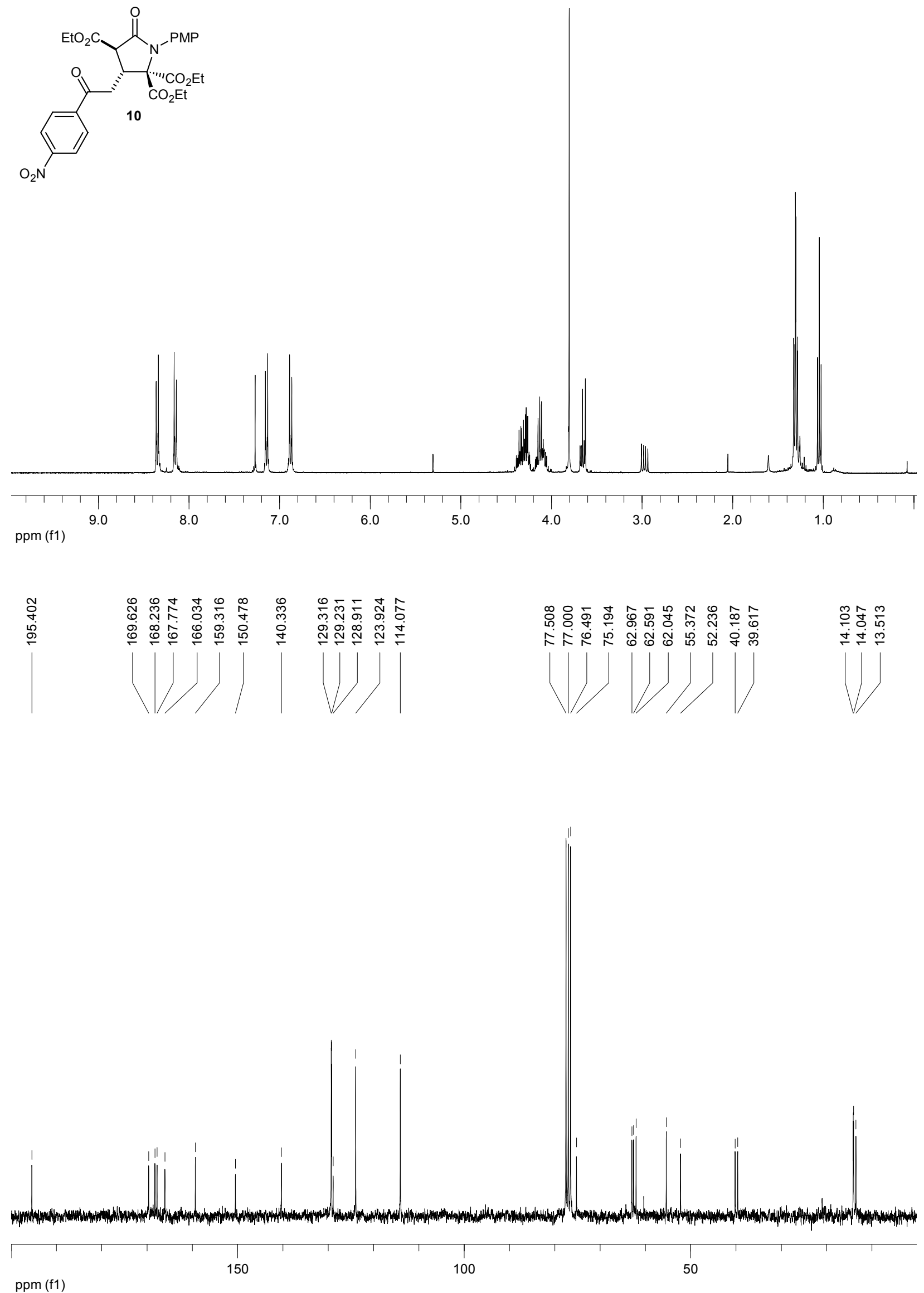

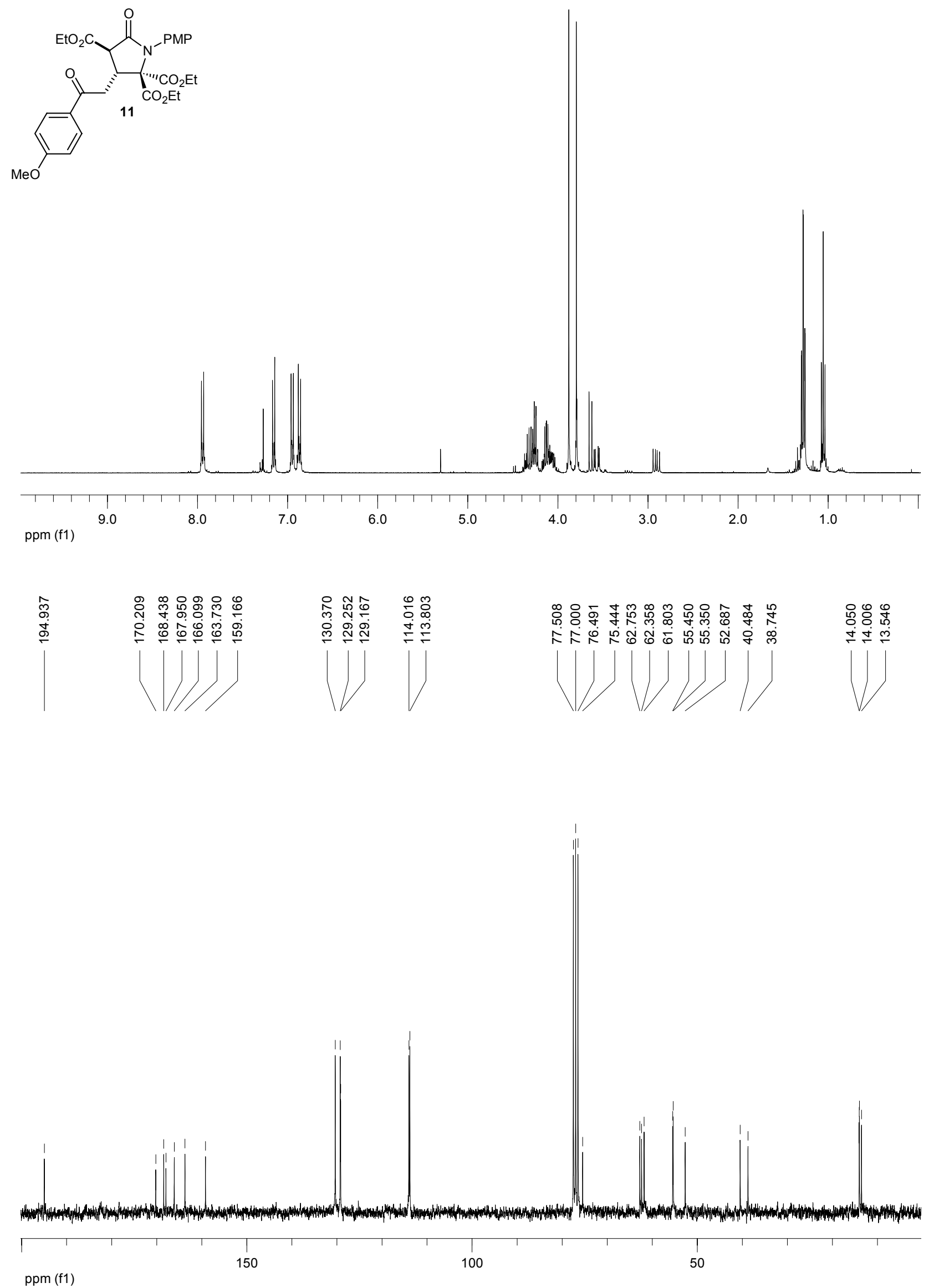

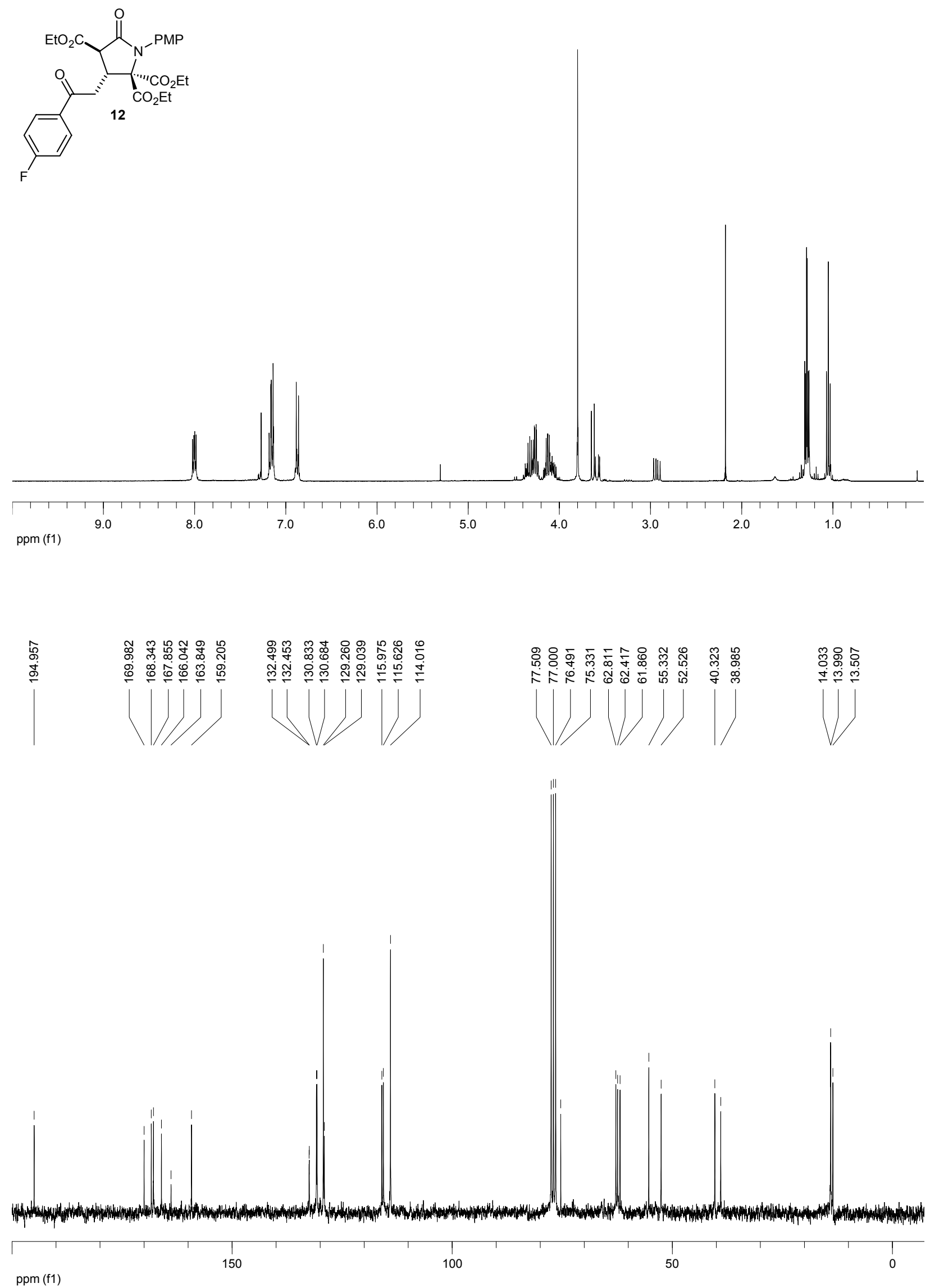

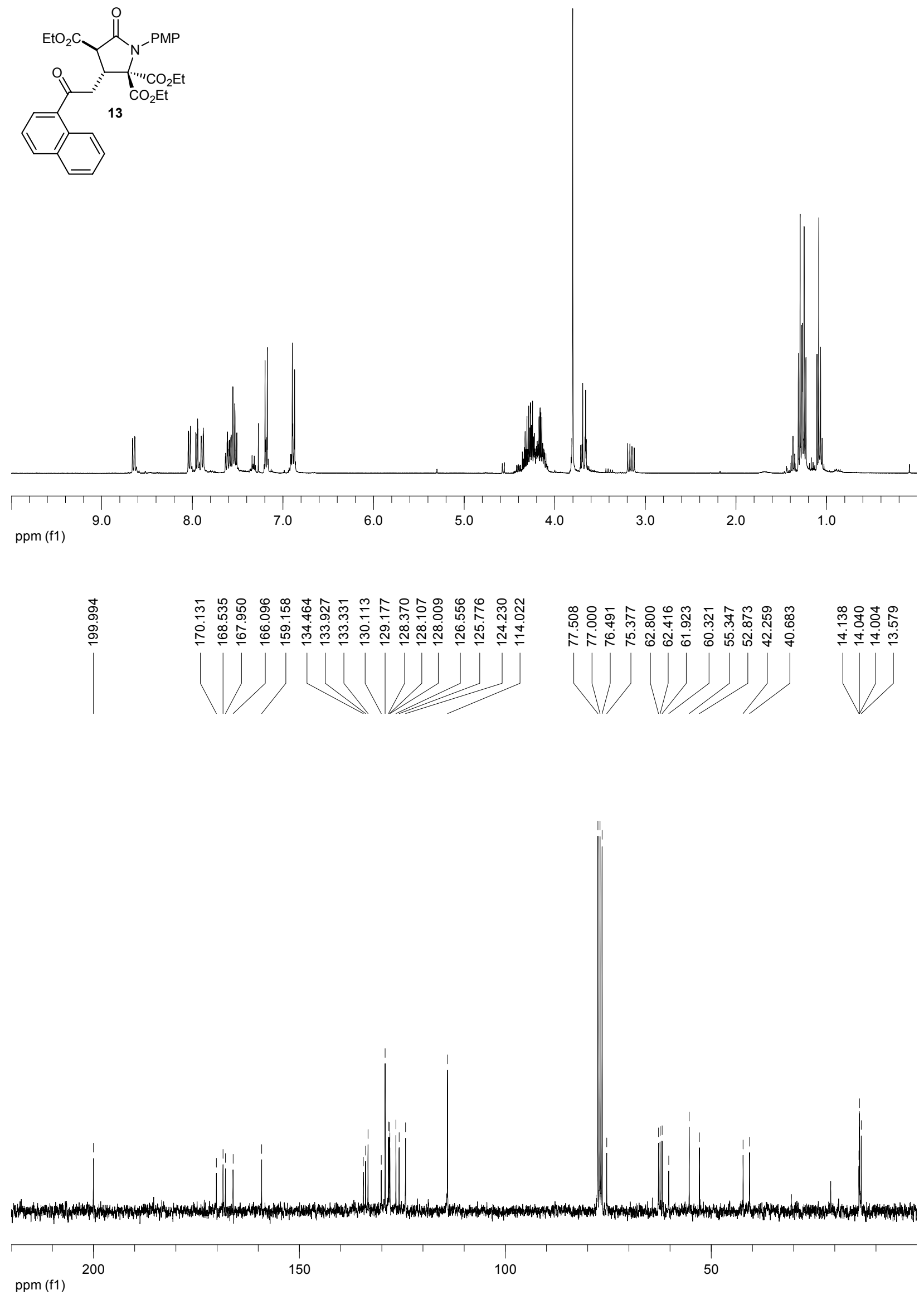

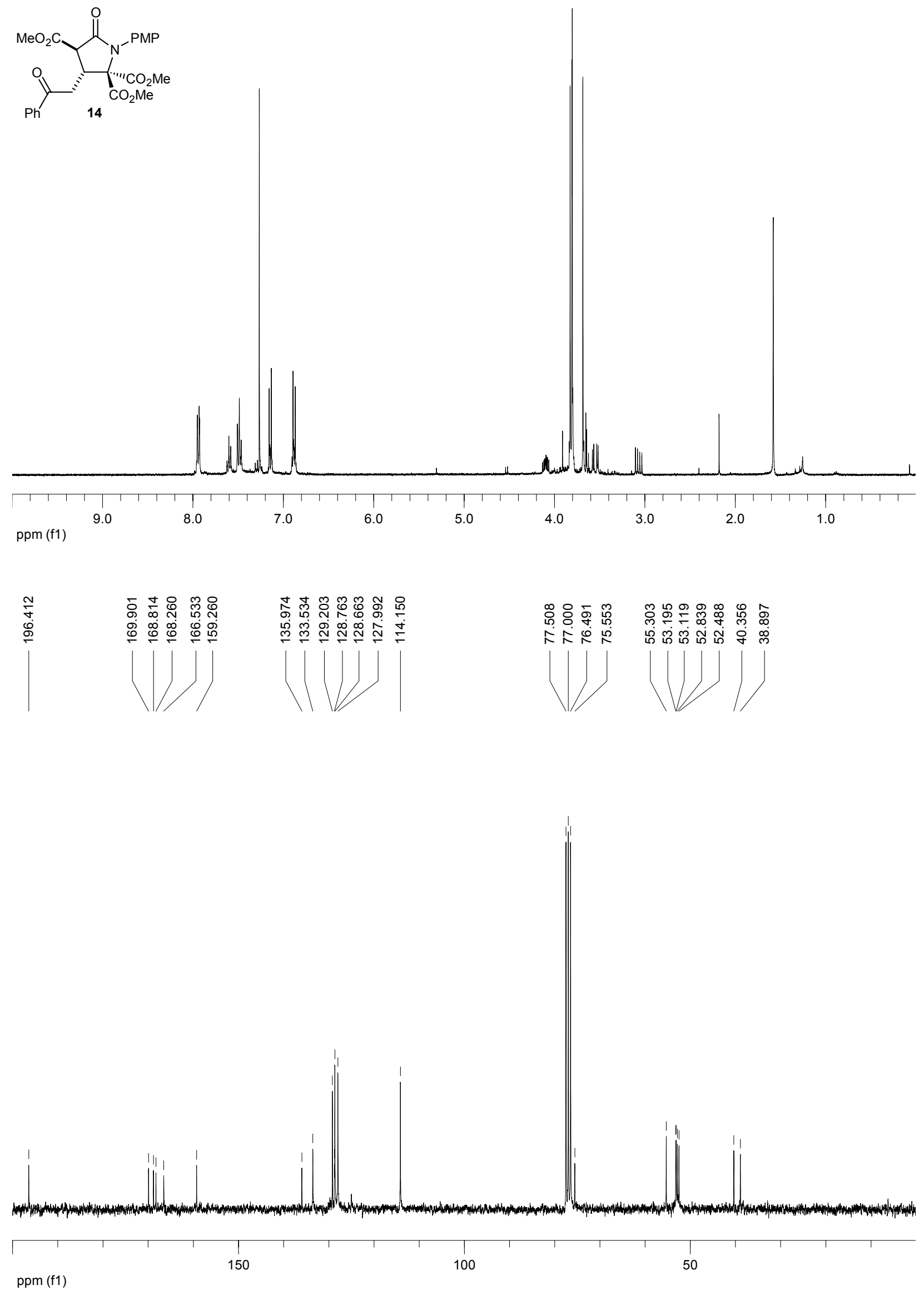


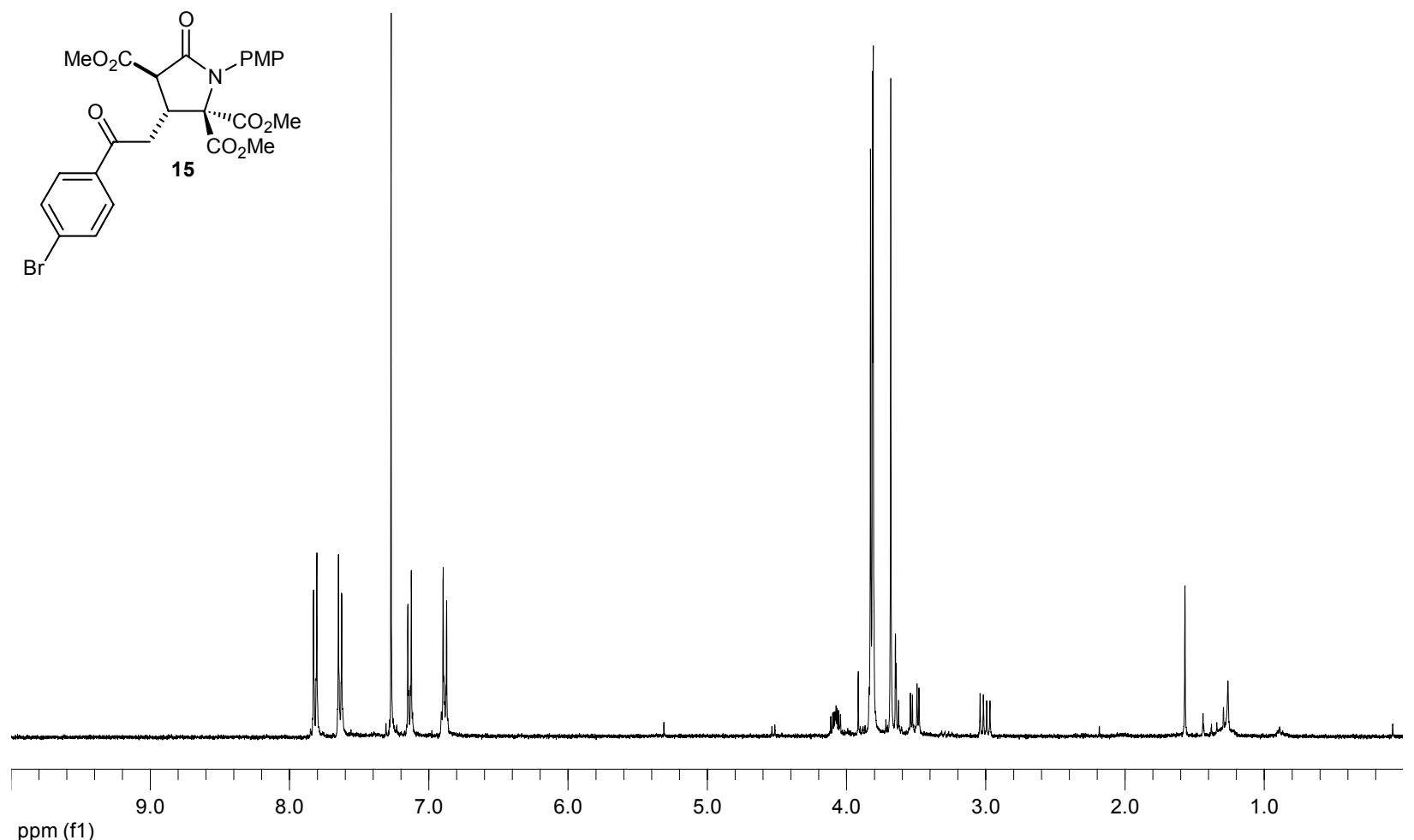

ppm (f1)

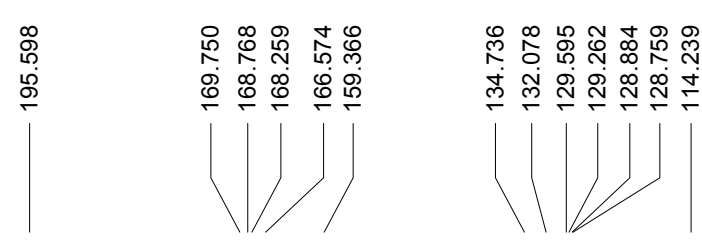

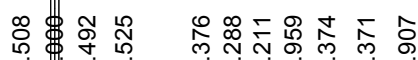

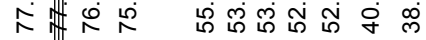
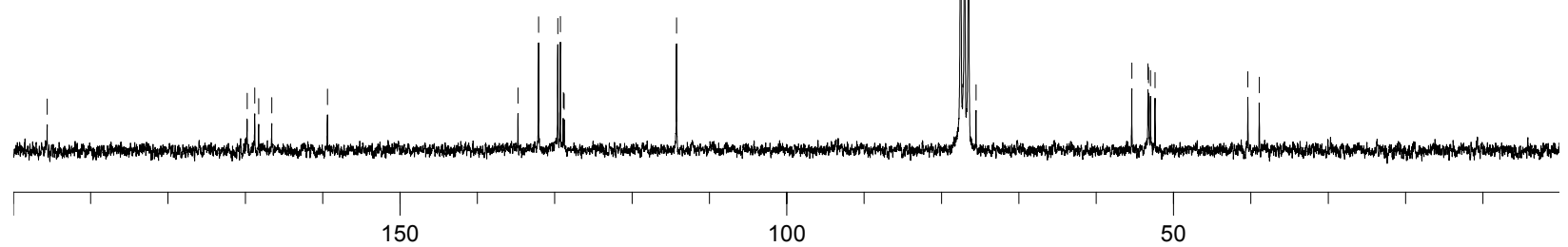

ppm (f1) 

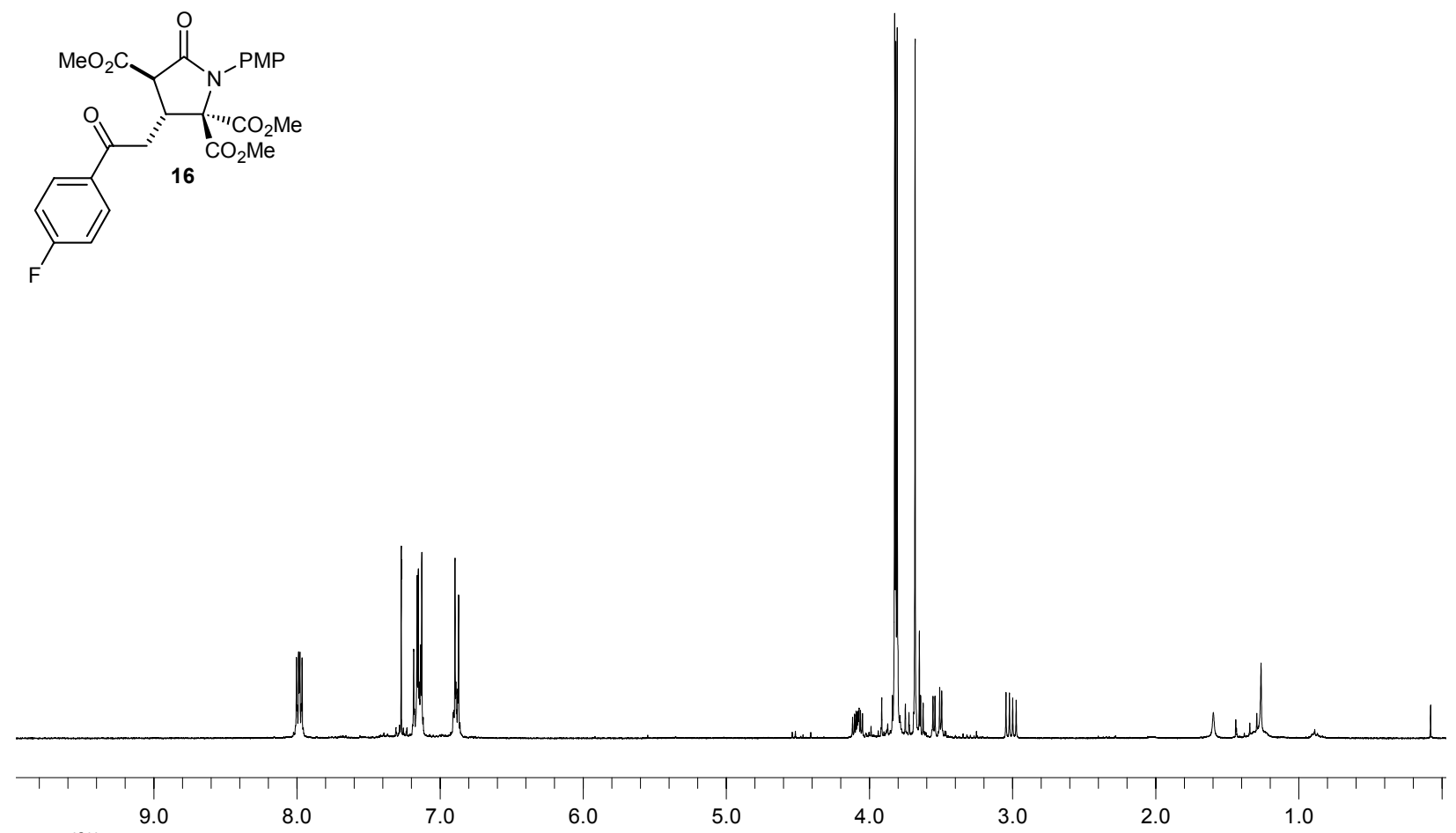

ppm (f1)
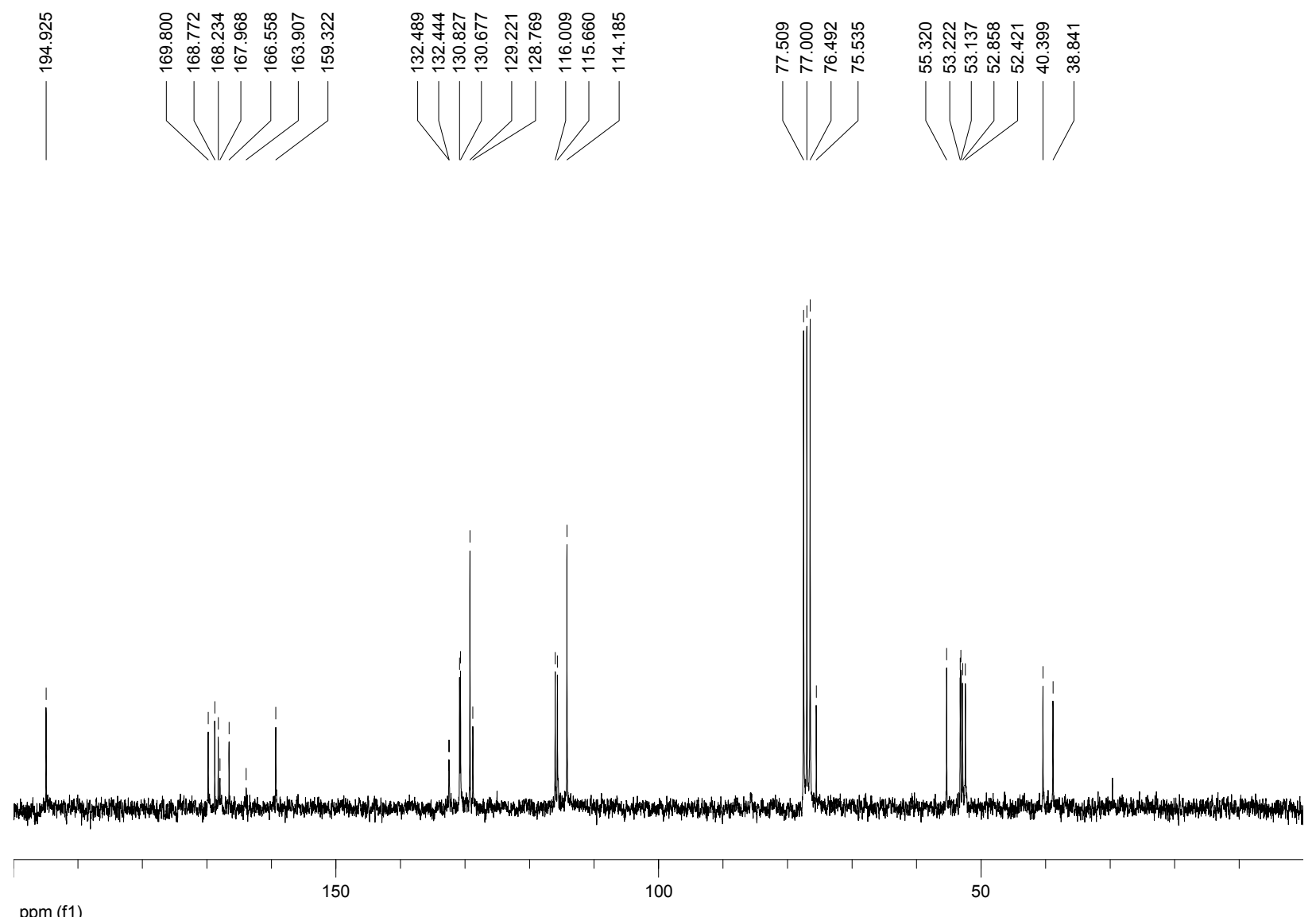


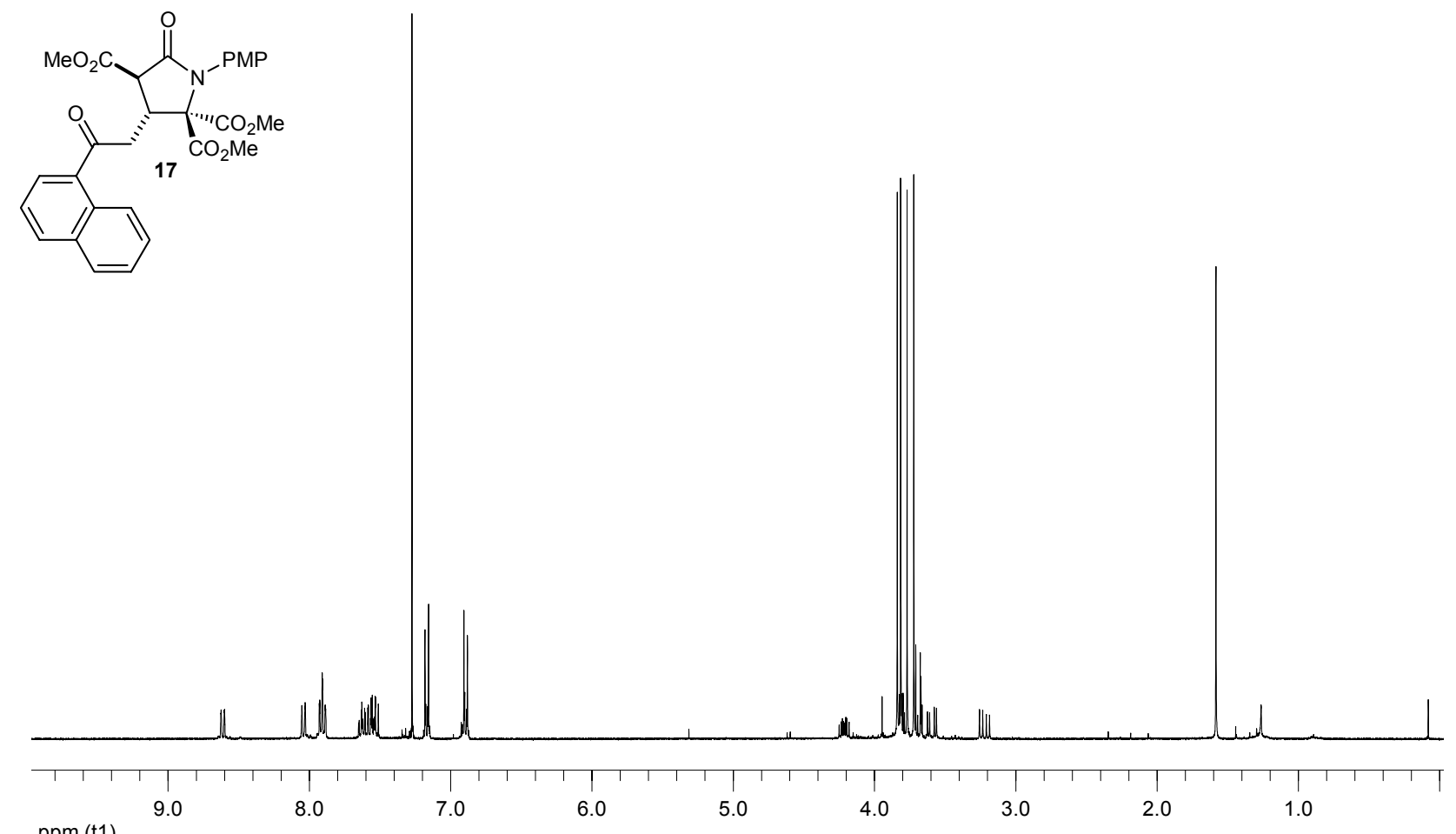

ppm (t1)
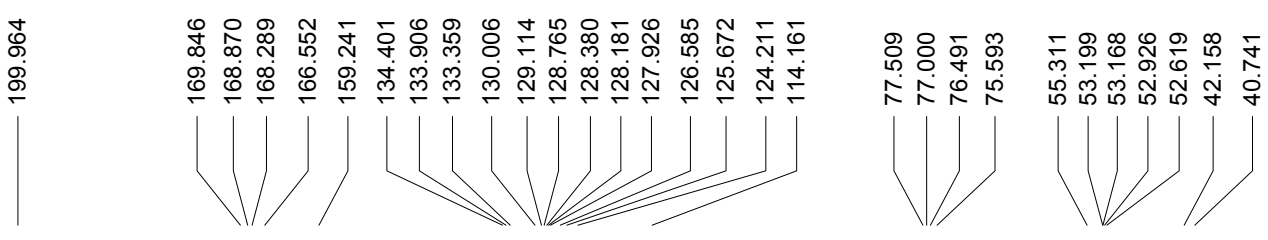

रำ

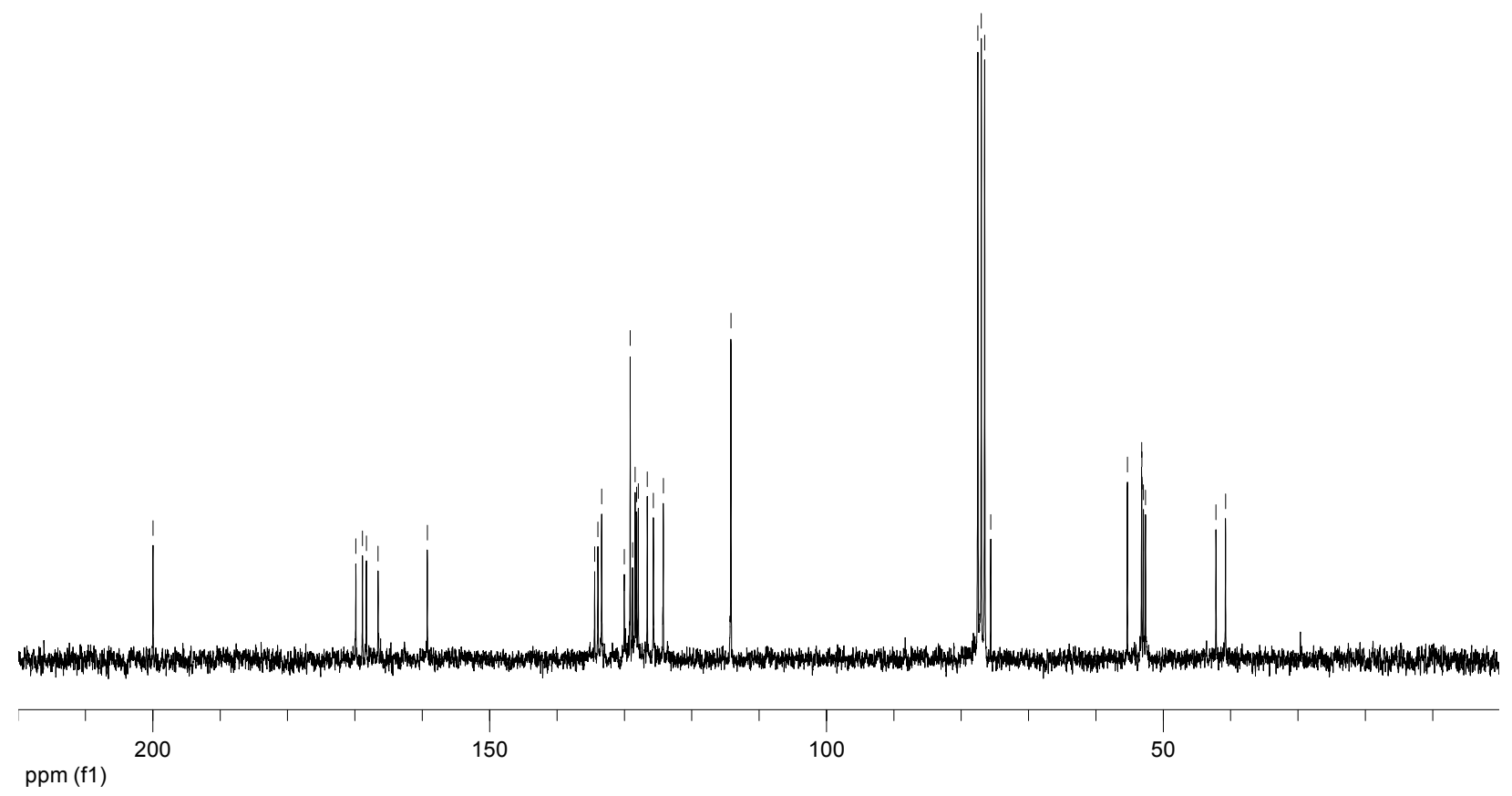




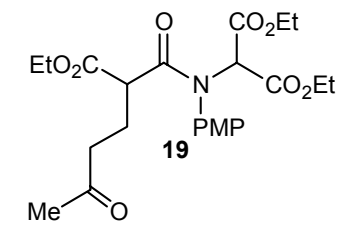

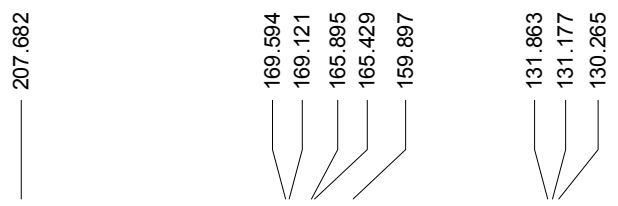
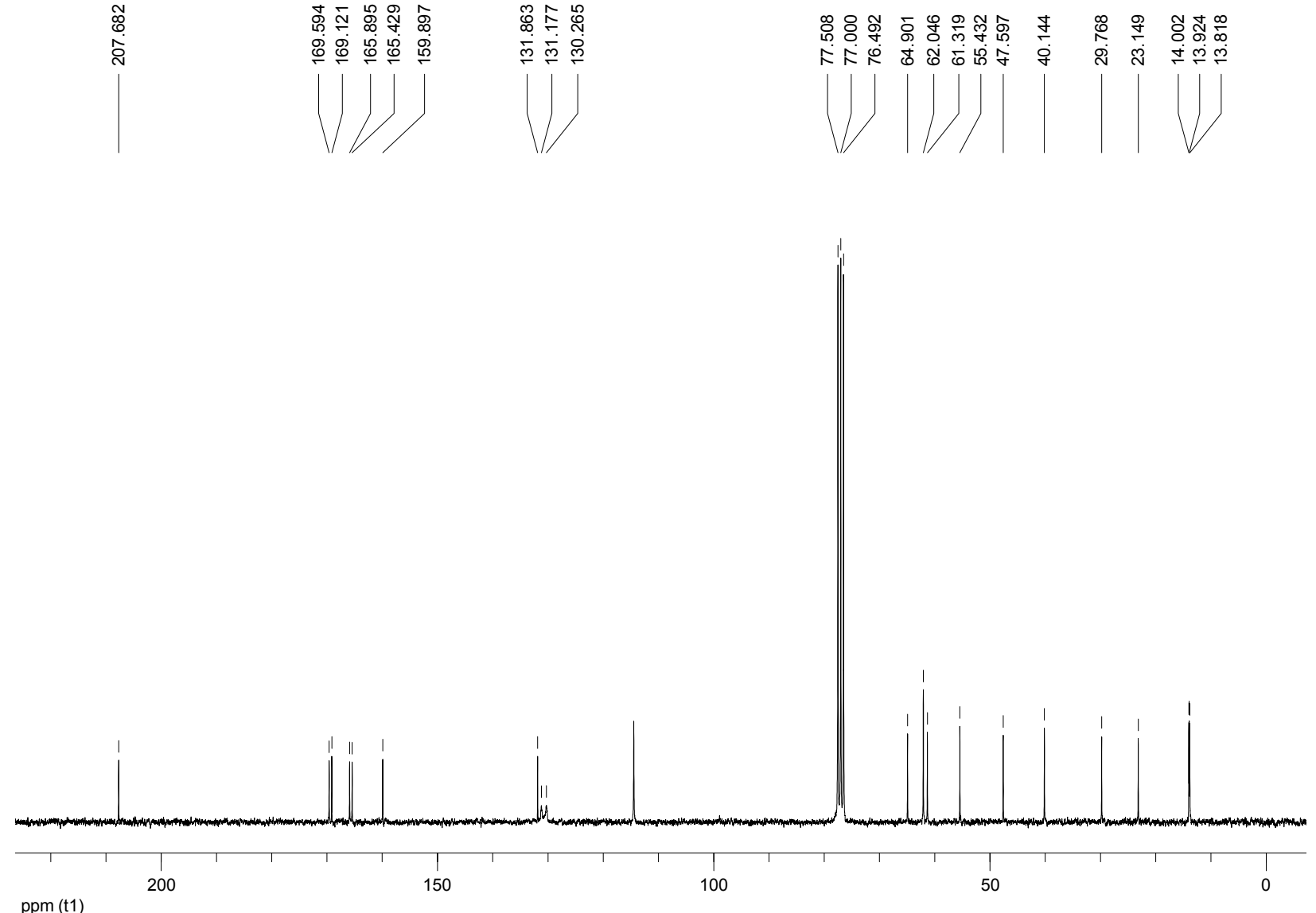

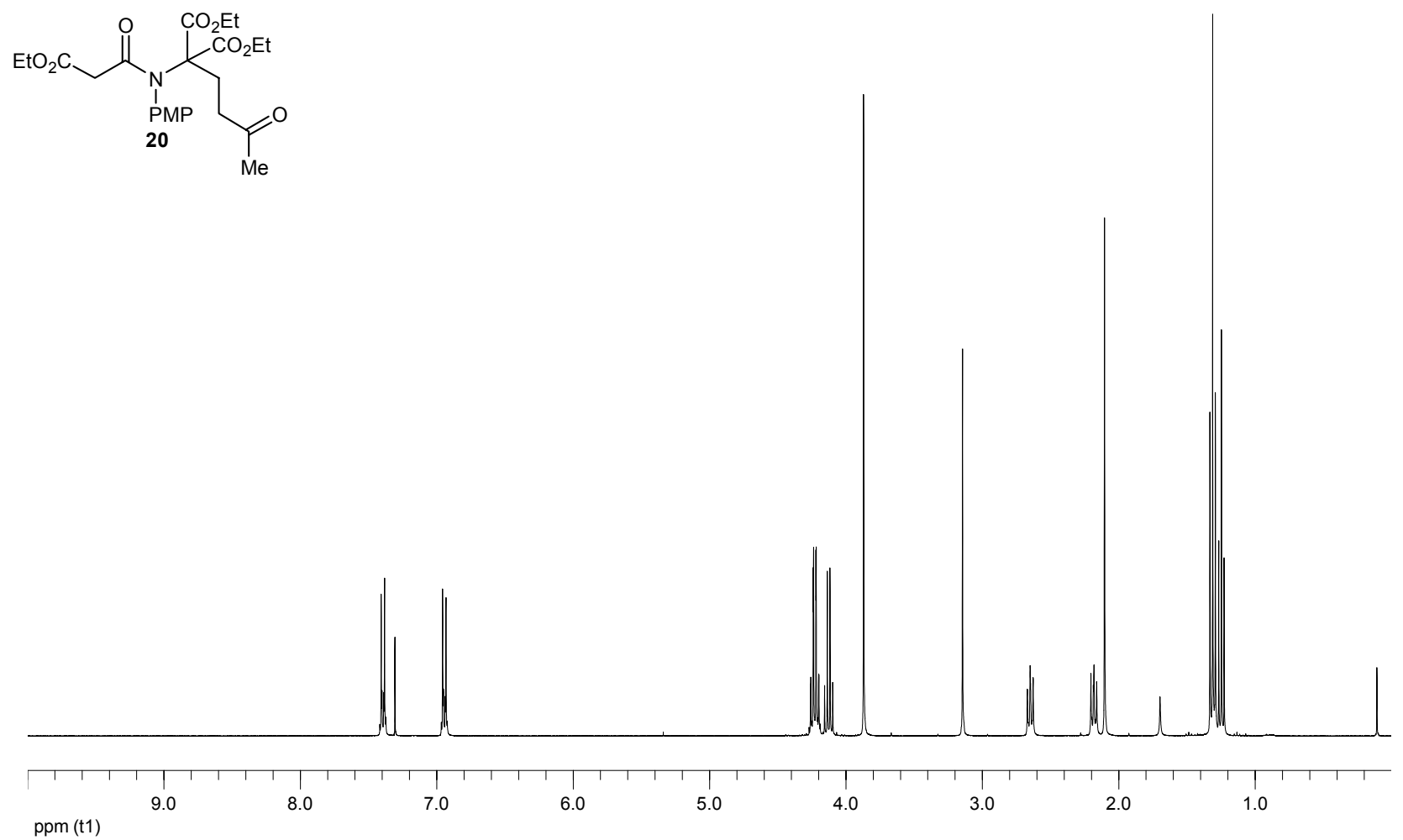

ppm (t1)

$\overbrace{\substack{\infty \\ 0}}^{\substack{0 \\ 0}}$
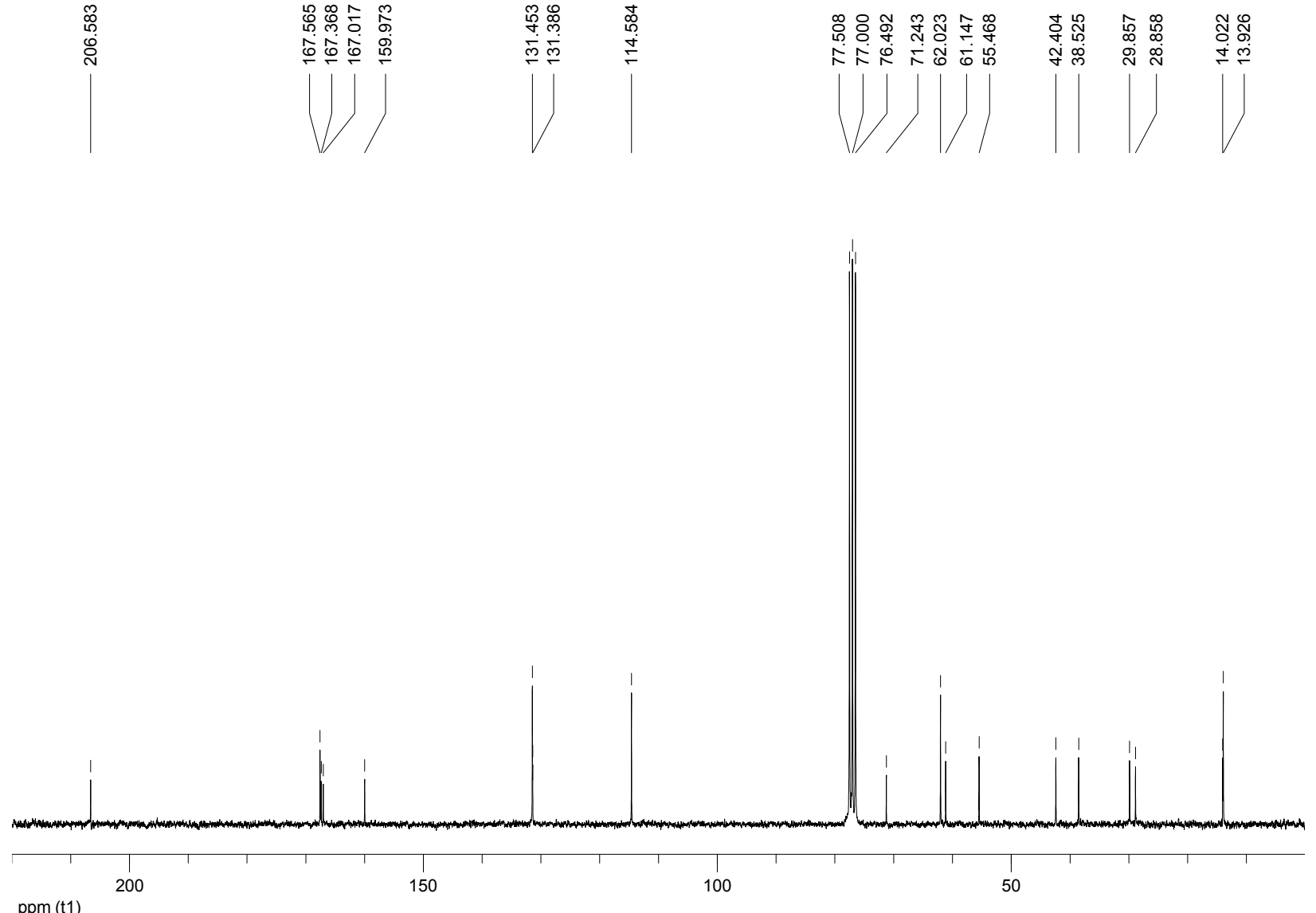

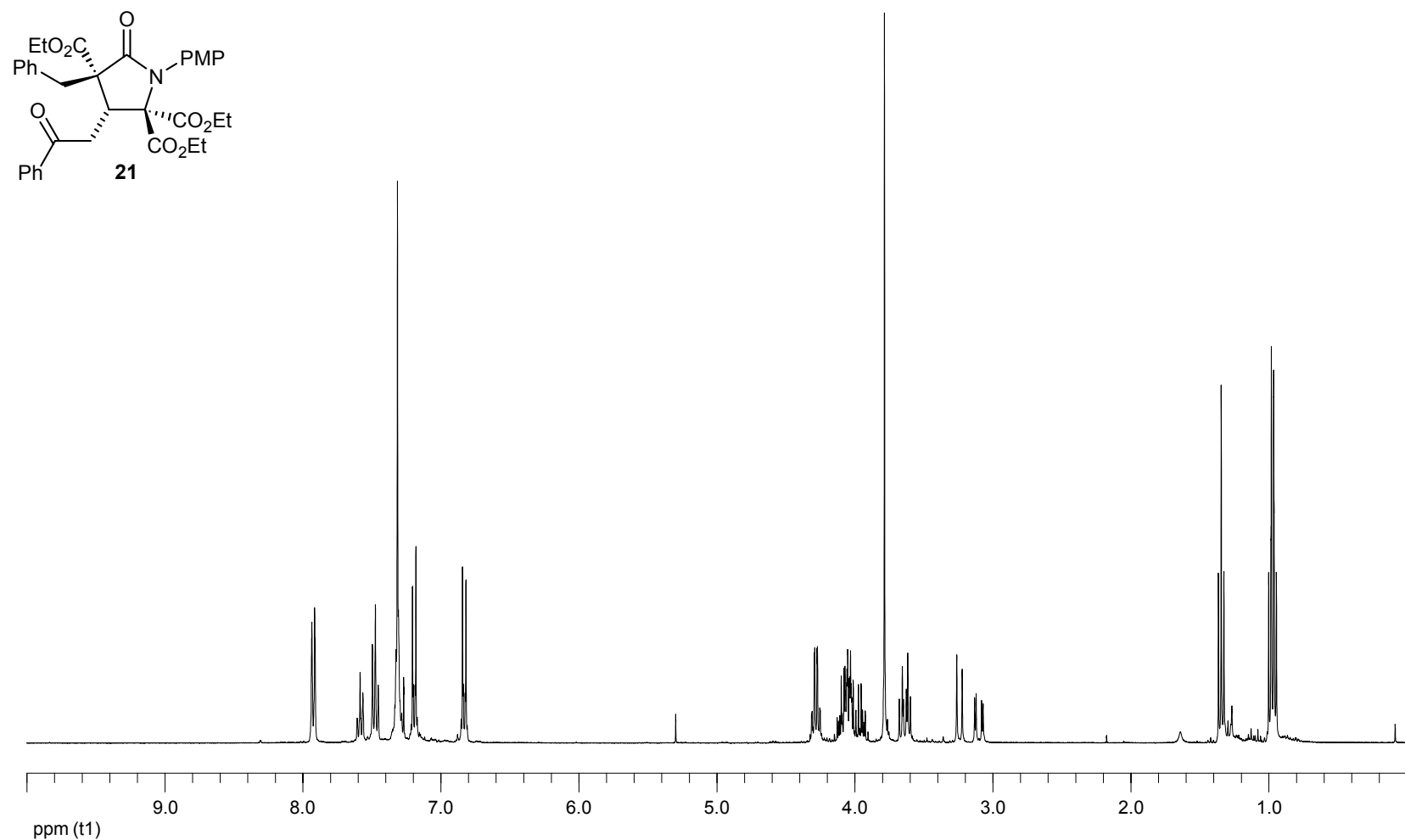

ppm (t1)

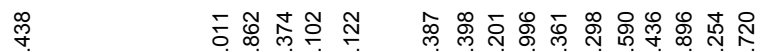

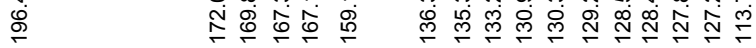
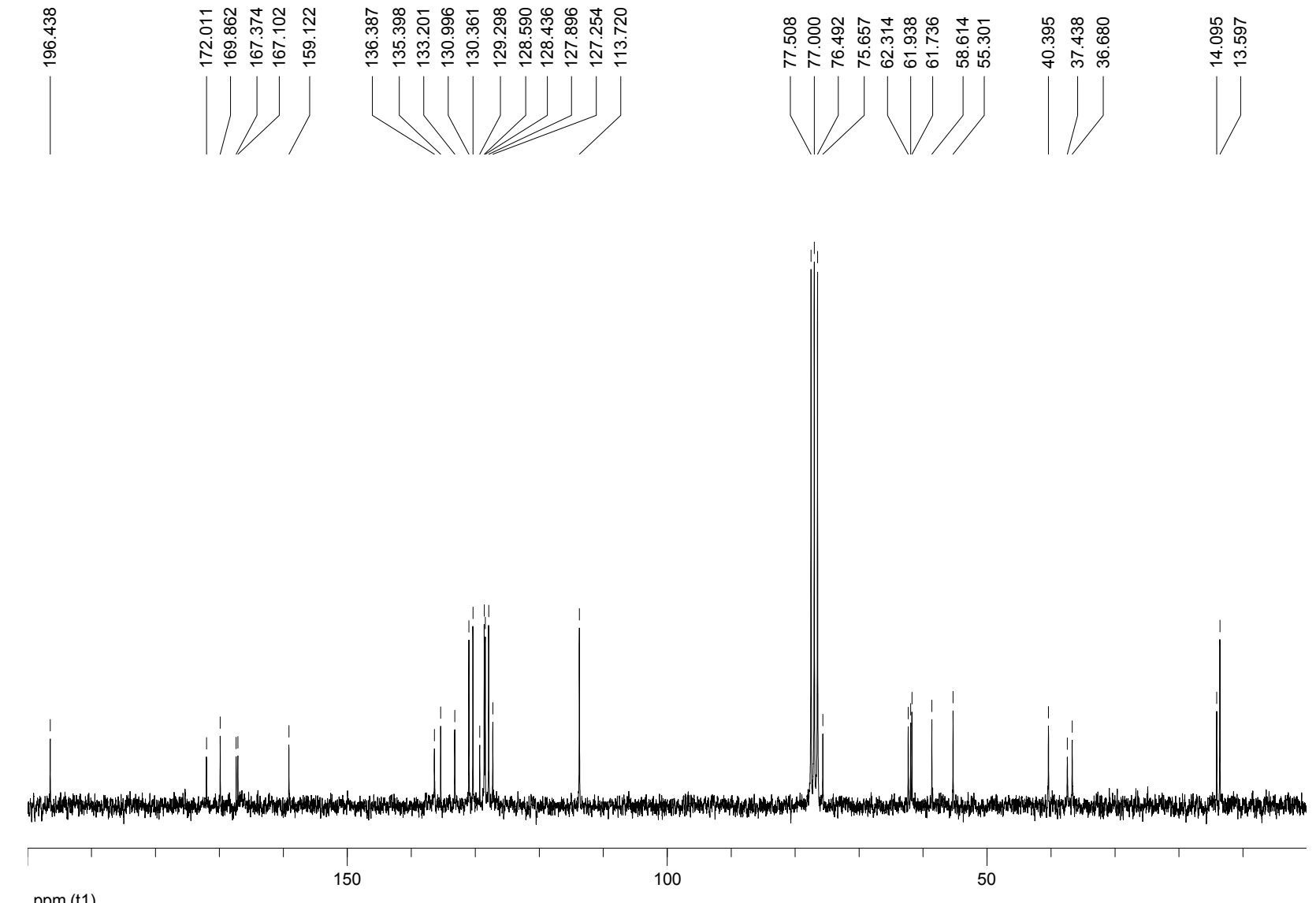

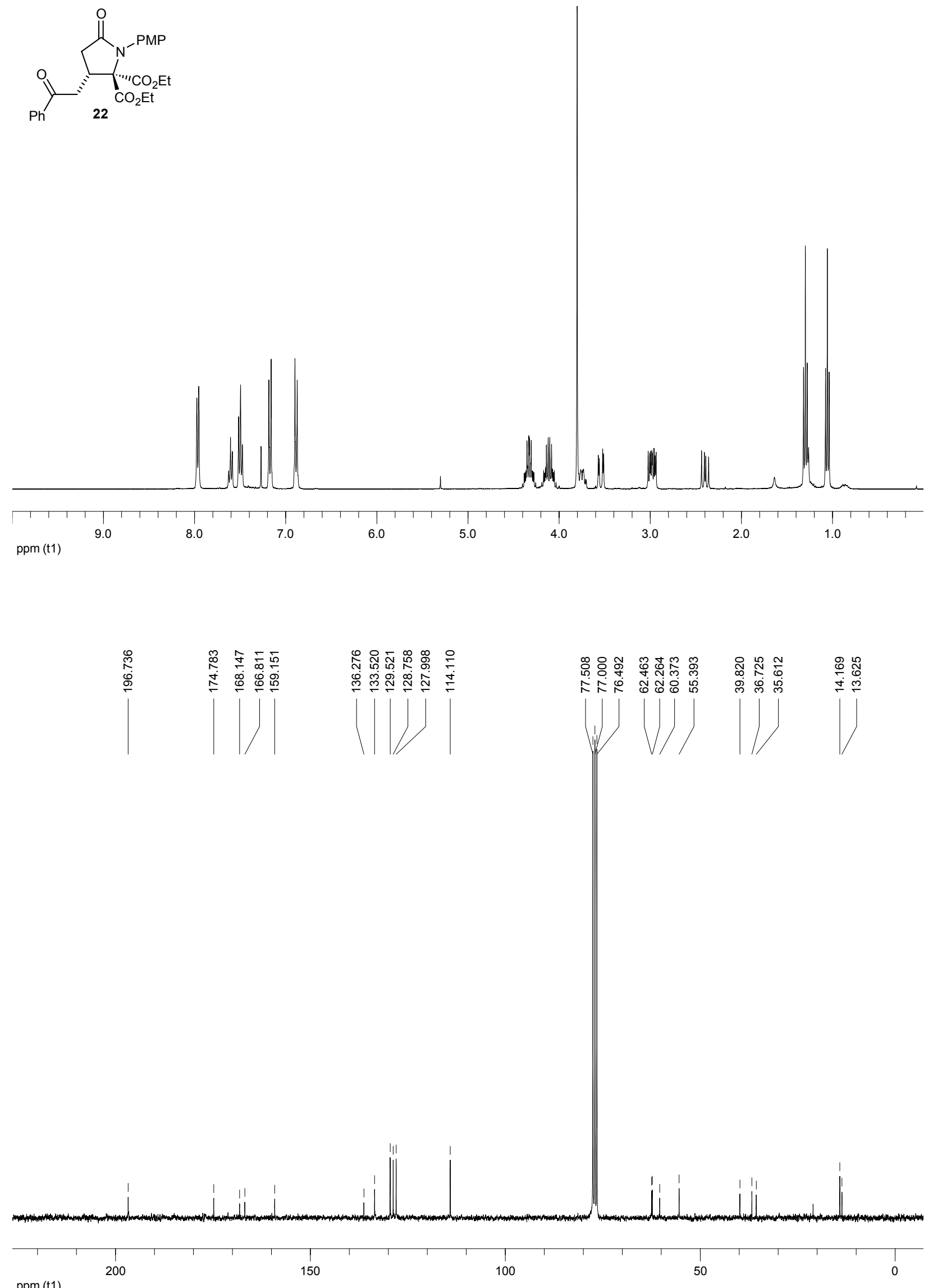

ppm (t1) 